Technical Memorandum 86058

\title{
Conservation Laws of Wave Action and Potential Enstrophy for Rossby Waves in a Stratified Atmosphere
}

David M. Straus

December 1983

Laboratory for Atmospheric Sciences Global Modeling and Simulation Branch

National Aeronautics and

Space Administration

Goddard Space Flight Center

Greenbelt, Maryland 20771 
Conservation Laws of Wave Action and Potential Enstrophy

for Rossby Waves in a Stratified Atmosphere

\author{
David M. Straus \\ Laboratory for Atmospheric Sciences \\ NASA/Goddard Space Flight Center \\ Greenbelt, MD 20771
}

December 1983 
TABLE OF CONTENTS

Preface $\ldots \ldots \ldots \ldots \ldots \ldots \ldots \ldots \ldots \ldots \ldots \ldots \ldots \ldots \ldots \ldots \ldots \ldots \ldots \ldots \ldots \ldots \ldots$

1. Introduction $\ldots \ldots \ldots \ldots \ldots \ldots \ldots \ldots \ldots \ldots \ldots \ldots \ldots \ldots \ldots \ldots \ldots \ldots \ldots \ldots \ldots$

2. The Elementary Theory of Rossby Waves $\ldots \ldots \ldots \ldots \ldots \ldots \ldots \ldots \ldots \ldots \ldots$

3. The Kinematic Theory of Wavetrains $\ldots \ldots \ldots \ldots \ldots \ldots \ldots \ldots \ldots \ldots \ldots$

4. Conservation Laws for Wave Energy, Wave Action and Wave Enstrophy in a Slowly Varying Mean Flow ............................... 17

5. Connection with Wave Activity, Potential Vorticity and the Eliassen-Palm Flux; Alternative Derivations .................. 29

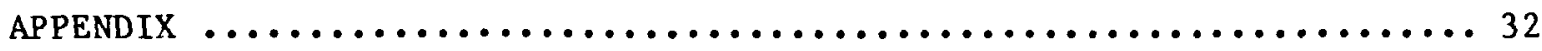




\section{Preface}

The purpose of this memorandum is to discuss the evolution of wave energy, enstrophy and action for atmospheric Rossby waves in a variable mean flow. The presentation is theoretical, but does not represent original research; rather, it is pedagogic in nature. The work of a number of people has been drawn together into a unified account, with much of the algebra implicit in previous work made explicit here. The central results are the conservation laws given in Equations $(4.24),(4.33),(4.34)$ and $(5.10)$.

It is a pleasure to thank David Andrews, who introduced the author to the concept of wave action and stimulated his interest in many of the concepts treated in this memorandum. Useful discussions with Brian Hoskins and Eugenia Kalnay are also acknowledged. Thanks also go to Ms. Mary Ann Wells, who did such an excellent job of typing the manuscript. 


\section{Introduction}

Conservation laws play an important role in geophysical fluid dynamics, for they yield strong insights into how flows are dynamically constrained. Such laws may apply to the fluid system as a whole, or to wave-like excitations of the fluid. The purpose of this article is to present a comprehensive development of the conservation laws of energy, enstrophy and wave action for atmospheric Rossby waves. This work is not new or original, but synthesizes and expands the published derviations of many others (Young and Rhines, 1980; Lighthi11, 1978; Edmon et al., 1980) into what is hopefully a clear and complete picture of how these conservation laws arise for Rossby waves, and what their limitations are. The powerful variational methods developed by Bretherton and Garrett (1968), Hayes (1970) and Andrews and McIntyre (1978) have not been used, as they are excessively abstract for the current purpose.

To motivate the study of wave action and wave enstrophy, let us consider the more familiar notion of wave energy. Keeping in mind that we are dealing with linear systems, it is usually the case that wave energy in not conserved. For unstable waves, this is to be expected, since the terms representing the conversion of energy (kinetic or available potential) from the basic state to the wave will appear as a source term for the wave energy. However, conservation of wave energy also fails to hold for linear Rossby waves propagating in a slowly varing mean flow. There the local amplitude and energy density of the wave increase as the wave propagates towards larger values of the mean $f$ low $\bar{u}$, even though the wave does not draw energy from the mean flow (1.e. the wave is neutral). Since Rossby waves play a role in a variety of adjustment problems, including the mediation of tropical-extratropical interactions (see, for example, Hoskins and Karoly, 1981), it is of interest to find a wave quantity which is conserved in a varlable mean flow. The two central results of this article 
are that: (1) In the absence of longitudinal (or $x$ ) variations in the mean flow, both wave action and wave enstrophy are conserved locally; and (2) For arbitrary slow variations in the mean flow, only wave enstrophy is conserved locally. The directional dependence of result (1) above is due to the fact that only meridional (or $y$ ) gradients of the planetary vorticity $f$ are present; the anisotropic nature of the wave appears already in the fundamental dispersion relation.

The basic strategy is to develop the elementary theory of a Rossby wave in a constant basic state $\bar{u}$, and then to assume that even when $\bar{u}$ is variable, the wave dispersion and wave energy density are related to the local value of $\bar{u}$ and the local wave amplitude as in the elementary theory. The kinematic theory of Lighthill (1978) is then used to describe the rates of change of frequency and three dimensional wavenumber. This approach does not explicity make use of WKB theory (as does the derivation of Young and Rhines, 1980), although the spirit of the calculation is much the same. We also do not follow the approach of Hoskins et a1.(1983), who derive the local conservation law for wave potentia1 enstrophy (our eq. (4.34)) because this approach does not make explicit how the frequency and wavenumber vector change in response to a slowly changing mean wind.

It is perhaps important to point out that the local approach used in this article is not valid for unstable waves, since growth rates are generally determined by the - configuration- of-the-mean- flow- $^{-}$- over- the whole domain- and -so depend on $\bar{u}$ in a non-local fashion. Thus, in order to study the form of the source terms that would arise (in the unstable case) in the conservation laws for wave action and wave enstrophy, one would have to either assume a vanishingly small growth rate, or seek an approach different than the one presented here. Section 2 presents the governing equations and the elementary theory of a Rossby wave in a constant mean flow u. The kinematical derivation for the 
rates of change of wavenumber and frequency for a slowly varying wave train is presented in Section 3. Using the results of Section 3, the elementary theory of Section 2 is generalized in Section 4 to the case of a slowly varying mean flow, leading to the derivation of the conservation laws for wave enstrophy and wave action. Wave action is related to wave activity and the Eliassen-Palm flux vector in Section 5 . 


\section{The Elementary Theory of Rossby Waves}

2a. The governing equations .

The governing equations are those of quasigeostrophic theory on a beta plane. They are most easily derived from scaling considerations when altitude $\left(z^{*}\right)$ rather than pressure $(p)$ is used as the vertical coordinate (e.g. Charney and Stern, 1962). Furthermore, Rossby waves are more easily treated using $z^{*}$ as a vertical coordinate. On the other hand, the advantages of using $p$ as a vertical coordinate are well known. In this article we follow Palmer (1982), and retain the advantages of both systems by using log (pressure) coordinates:

$$
z=-H \ln \left(p / p_{o}\right)
$$

where $\mathrm{p}_{\mathrm{O}}=1000 \mathrm{mb}$ is a reference pressure and the scale height $\mathrm{H}$ is a constant. The quasigeostrophic equations on a beta plane are derived in $z$ coordinates in the Appendix. They take the form of the conservation of potential vorticity following the horizontal motion of the flow:

$$
\left(\frac{\partial}{\partial t}-\frac{\partial \psi}{\partial y} \frac{\partial}{\partial x}+\frac{\partial \psi}{\partial x} \frac{\partial}{\partial y}\right) q=0
$$

where the potential vorticity $\mathrm{q}$ is given by:

$$
q=f_{o}+B y+\nabla_{2}^{2} \psi+\frac{f_{o}^{2} H}{R S} e^{z / H} \frac{\partial}{\partial z}\left(e^{-z / H} \frac{\partial \psi}{\partial z}\right)
$$

Here $\psi$ is the geostrophic streamfunction, defined so that $u=-\frac{\partial \psi}{\partial y}, v=\frac{\partial \psi}{\partial x}$, with $u$ being the eastward and $v$ the northward velocity, and $\nabla_{2}^{2}$ is the horizontal Laplacian operator $\left(\frac{\partial^{2}}{\partial \mathrm{x}^{2}}+\frac{\partial^{2}}{\partial \mathrm{y}^{2}}\right)$. Also, $\mathrm{f}_{\mathrm{o}}=2 \Omega \sin \phi_{0}$, where $\Omega$ is the angular rotation rate of the earth and $\phi_{0}$ the central latitude of the beta plane, $\beta=\left.\frac{d f}{d y}\right|_{\phi_{O}}$, where $y$ is defined by $d y=a d \phi$, a being the earth's radius, $f=2 \Omega \sin \phi$, $R$ is the gas constant, and $s=\left(\frac{p}{p_{0}}\right)^{k} \frac{d \theta}{d z}, k=R / C_{p}, C_{p}$ being the specific heat at 
constant pressure, and $\theta_{S}=\theta_{S}(z)$ is the reference state potential temperature (Charney 1947: Charney and Stern, 1962). In deriving (2.3) it has been assumed that $S$ is constant in the vertical. We shall make that assumption throughout this article.

It is useful to relate the parameter $\frac{\mathrm{f}_{\mathrm{o}}{ }^{2 \mathrm{H}}}{\mathrm{RS}}$ appearing in (2.3) to the BruntVäisälä frequency $N_{s}$, given by $N_{s}{ }^{2}=\frac{g}{\theta_{s}} \frac{d \theta_{s}}{d z^{*}}$, where $z^{*}$ is altitude, as before. Since $\frac{\mathrm{d} \theta}{\mathrm{dz}} \mathrm{s}, \frac{\mathrm{d} \theta}{\mathrm{dp}} \mathrm{s}$ and $\frac{\mathrm{d} \theta}{\mathrm{dz}} \mathrm{s}$ depend only on the reference state,

$$
\frac{d \theta_{s}}{d z^{*}}=\frac{d \theta_{s}}{d p} \frac{d p_{s}}{d z^{*}}=-\frac{d \theta_{s}}{d p} \rho_{s} g=-\frac{d \theta_{s}}{d p} \frac{p g}{R T_{s}},
$$

where $\frac{d \theta_{s}}{d p}$ (written with $p$ as an independent coordinate) should be written as $\frac{\mathrm{d} \theta}{\mathrm{dp}_{\mathrm{S}}} \mathrm{if} \mathrm{z}^{*}$ is the independent coordinate. Similarly p apearing in the above formula should be understood as $p_{S}$ in the $z^{*}$ system. We have used the equation of state of an ideal gas, $p=\rho R T$. From this, $N_{s}^{2}=\frac{-p g^{2}}{R_{s} \theta_{s}} \frac{d \theta_{s}}{d p}$. Introducing $H_{s}=\frac{R T_{s}}{g}$, the scale height of an isothermal atmosphere with temperature $\mathrm{T}_{\mathbf{S}}$

$$
\mathbf{N}_{\mathbf{S}}{ }^{2}=\frac{-\mathrm{pg}}{\theta_{\mathbf{S}} \mathrm{H}_{\mathbf{S}}} \frac{\mathrm{d} \theta_{\mathbf{S}}}{\mathrm{dp}}=\frac{\mathrm{gH}}{\theta_{\mathbf{S}} \mathrm{H}_{\mathbf{S}}} \frac{\mathrm{d} \theta_{\mathbf{s}}}{\mathrm{dz}}=\frac{\mathrm{gH}}{\mathrm{H}_{\mathbf{S}} \mathrm{T}_{\mathbf{S}}} \mathrm{S},
$$

so that $\mathrm{S}=\frac{\mathrm{H}_{\mathrm{s}} \mathrm{T}_{\mathrm{s}}}{\mathrm{gH}} \mathrm{N}_{\mathrm{s}}^{2}$

and

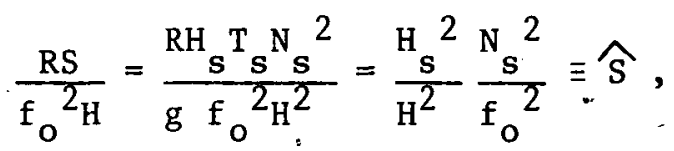

where equation (2.5) defines $\hat{\mathrm{S}}$. This quantity will appear in many of the subsequent equations in this article, and is closly analagous to the parameter S introduced by Pedlosky (1979, p. 334), who developed the equations in $z^{*}$ coordinates .

2b. Linearization: The Prototype Rossby wave Equation (2.2) is linearized about a basic state (denoted by an overbar): 


$$
\begin{aligned}
& \mathrm{q}=\bar{q}+\mathrm{q}^{-}, \text {and } \\
& \psi=\bar{\psi}+\psi^{\prime} \text {. }
\end{aligned}
$$

Here $\bar{q}$ and $\bar{\psi}$ can be functions of $(x, y, z)$ but not time $t$, whereas the perturbations $\mathrm{q}^{-}$and $\psi^{-}$are functions of $(x, y, z, t)$. Note that

$$
\begin{aligned}
& \overline{\mathrm{q}}=\mathrm{f}_{\mathrm{o}}+B \mathrm{y}+\nabla_{2}^{2} \bar{\psi}+\frac{1}{\widehat{\mathrm{s}}} \mathrm{e}^{z / H} \frac{\partial}{\partial z}\left(\mathrm{e}^{-\mathrm{z} / \mathrm{H}} \frac{\partial \bar{\psi}}{\partial z}\right), \\
& \mathrm{q}^{\prime}=\quad \nabla_{2}^{2} \psi^{-}+\frac{1}{\widehat{\mathrm{S}}} \mathrm{e}^{z / H} \frac{\partial}{\partial z}\left(\mathrm{e}^{-\mathrm{z} / \mathrm{H}} \frac{\partial \psi^{\prime}}{\partial z}\right) .
\end{aligned}
$$

The most general linear form of $(2.2)$ is

$$
\frac{\partial}{\partial t} q^{\prime}+\bar{u} \frac{\partial q^{\prime}}{\partial x}+u^{\prime} \frac{\partial \bar{q}}{\partial x}+\bar{v} \frac{\partial q^{\prime}}{\partial y}+v^{\prime} \frac{\partial \bar{q}}{\partial y}=0
$$

We will return to (2.7) in Section 4 . The prototype Rossby wave is obtained in this section by setting

$$
\bar{\psi}=-\vec{u} y,
$$

where $\bar{u}$ is assumed constant. Thus $\bar{v}=0$, and from $(2.6 a) \bar{q}=f_{o}+\beta y$, so that $\frac{\partial \bar{q}}{\partial x}=0$ and $\frac{\partial \bar{q}}{\partial y}=\beta \cdot$ Equation $(2.7)$ becomes

$$
\frac{\partial q^{\prime}}{\partial t}+\bar{u} \frac{\partial q^{\prime}}{\partial x}+\beta v^{\prime}=0
$$

-We try a solution of the form

$$
\psi^{\prime}=\mathrm{Ae}^{z /(2 \mathrm{H})} \cos (\mathrm{kx}+\ell \mathrm{y}+\mathrm{mz}-\omega t) \equiv \mathrm{Ae}^{z /(2 \mathrm{H})} \cos (\alpha) .
$$

Here $A$ is the (constant) wave amplitude, $(k, l, m$ ) the (constant) wave vector, and $\omega$ the (constant) frequency.

From (2.6b) and (2.10) it is easy to show that 


$$
\begin{aligned}
q^{\prime} & =-\left[k^{2}+\ell^{2}+\frac{1}{\widehat{S}}\left(m^{2}+\frac{1}{4 H^{2}}\right)\right] A e^{z /(2 H)} \cos (\alpha) \\
& \equiv-K^{2} \mathrm{Ae}^{z /(2 H)} \cos (\alpha),
\end{aligned}
$$

which defines the three dimensional wavenumber

$$
\mathrm{K}^{2}=\mathrm{k}^{2}+\ell^{2}+\frac{1}{\mathrm{~S}}\left[\mathrm{~m}^{2}+\frac{1}{4 \mathrm{H}^{2}}\right] .
$$

Using (2.10) and (2.11) to evaluate each term in (2.9),

$$
\begin{aligned}
& \frac{\partial q^{\prime}}{\partial t}=-\omega \mathrm{K}^{2} \mathrm{Ae}^{\mathrm{z} /(2 \mathrm{H})} \sin (\alpha), \\
& \frac{\partial \mathrm{q}^{\prime}}{\partial \mathrm{x}}=+k \mathrm{~K}^{2} \mathrm{Ae}^{\mathrm{z} /(2 \mathrm{H})} \sin (\alpha),
\end{aligned}
$$

and

$$
v^{\prime}=\frac{\partial \psi^{\prime}}{\partial x}=-k \mathrm{Ae}^{z /(2 \mathrm{H})} \sin (\alpha)
$$

Equation (2.11) then gives the dispersion relation of a Rossby wave propagating in a constant mean flow:

$$
\omega=\overline{\mathrm{u}} \mathrm{k}-\frac{\beta \mathrm{k}}{\mathrm{k}^{2}}
$$

2c. Energy and Potential Enstrophy Density

The perturbation energy is defined as the sum of perturbation kinetic plus available potential energy. In ordinary pressure coordinates (Lorenz, 1960),

$$
\mathrm{E}_{\mathrm{TOT}}=\mathrm{E}_{\mathrm{K}}+\mathrm{E}_{\mathrm{A}} \text {, }
$$

with

$$
E_{K}=\iiint d x d y \frac{d p}{g} \frac{1}{2}\left[\left(\frac{\partial \psi^{-}}{\partial x}\right)^{2}+\left(\frac{\partial \psi^{-}}{\partial y}\right)^{2}\right]
$$

and

$$
E_{A}=\iiint d x d y \frac{d p}{g} \frac{R\left(T^{-}\right)^{2}}{2 \widetilde{s}},
$$

where

$$
\widetilde{s}=-p\left(\frac{p}{p_{0}}\right)^{k d \theta_{s}} \frac{\text { dp }}{d p} \text {. }
$$


Converting from $p$ to $z$ coordinates via (2.1),

$$
\mathrm{E}_{\text {TOT }}=\frac{\mathrm{P}_{\mathrm{O}}}{\mathrm{gH}} \iiint \mathrm{dxdydz} \mathrm{e}^{-z / H}\left\{\frac{1}{2}\left[\left(\frac{\partial \psi^{-}}{\partial \mathrm{x}}\right)^{2}+\left(\frac{\partial \psi^{-}}{\partial \mathrm{y}}\right)^{2}\right]+\frac{1}{2} \frac{\mathrm{R}\left(\mathrm{T}^{-}\right)^{2}}{\widetilde{\mathrm{S}}}\right\}
$$

Using the quasigeostrophic relationship $\mathrm{T}^{-}=\frac{\mathrm{f}_{0} \mathrm{H}^{\mathrm{H}}}{\mathrm{R}} \frac{\partial \psi^{-}}{\partial z}$ (see equation (A.10) in the appendix), and introducing $\mathrm{T}_{0} \equiv \frac{\mathrm{gH}}{\mathrm{R}}$ and $\rho_{\mathrm{o}} \equiv \frac{\mathrm{p}_{\mathrm{o}}}{\mathrm{gH}}=\frac{\mathrm{P}_{\mathrm{o}}}{\mathrm{RT}_{\mathrm{O}}}$, we have

$$
\mathrm{E}_{\text {TOT }}=\iiint \mathrm{d} x \mathrm{dy} \mathrm{dz} \rho_{\mathrm{o}} \mathrm{e}^{-\mathrm{z} / \mathrm{H}}\left\{\frac{1}{2}\left[\left(\frac{\partial \psi^{-}}{\partial \mathrm{x}}\right)^{2}+\left(\frac{\partial \psi^{-}}{\partial \mathrm{y}}\right)^{2}\right]+\frac{1}{\widehat{\mathrm{S}}}\left(\frac{\partial \psi^{-}}{\partial z}\right)^{2}\right\}
$$

The temperature $T_{O}$ is that which renders $\mathrm{gH} / \mathrm{R}$ a scale height for an isothermal atmosphere, while $\rho_{\circ}$ is an "equivalent" surface density.

From (2.14) we define an energy density (energy per unit mass) $\varepsilon$ as

$$
\varepsilon \equiv \frac{1}{2} e^{-z / H}\left[\left(\frac{\partial \psi^{-}}{\partial x}\right)^{2}+\left(\frac{\partial \psi^{-}}{\partial y}\right)^{2}+\frac{1}{\widehat{S}}\left(\frac{\partial \psi^{-}}{\partial z}\right)^{2}\right]
$$

Note that the energy density includes the exponential decrease of density with height. From (2.10),

$$
\begin{aligned}
E=\frac{1}{2} A^{2}\left\{\left(k^{2}+l^{2}\right) \sin ^{2}(\alpha)+\frac{1}{\widehat{S}}\left[\frac{1}{4 \mathrm{H}^{2}} \cos ^{2}(\alpha)\right.\right. & +\mathrm{m}^{2} \sin ^{2}(\alpha) \\
& \left.\left.-\frac{\mathrm{m}}{\mathrm{H}} \cos (\alpha) \sin (\alpha)\right]\right\}
\end{aligned}
$$

Averaging over one wave period

$$
E \equiv \frac{-1}{2 \pi} \int_{0}^{2 \pi} d \alpha \varepsilon=\frac{1}{4} A^{2}\left(k^{2}+l^{2}+\frac{1}{\widehat{S}}\left[\frac{1}{4 \mathrm{H}^{2}}+\mathrm{m}^{2}-\right]\right)=\frac{1}{4} \mathrm{~A}^{2} \mathrm{~K}^{2}
$$

The total perturbation potential enstrophy is

$$
\mathrm{P}_{\mathrm{TOT}}=\iiint \mathrm{dxdy} \frac{\mathrm{dp}}{\mathrm{g}}\left(\frac{1}{2} \mathrm{q}^{-2}\right)
$$

As with energy, this can be written as $P_{T O T}=\iiint d x d y d z \rho_{0} e^{-z / H}\left(\frac{1}{2} q^{-2}\right)$, leading 
to the definition of potential enstrophy density $\rho$ as

$$
\rho \equiv \mathrm{e}^{-\mathrm{z} / \mathrm{H}} \frac{1}{2} \mathrm{q}^{-2}
$$

Using (2.11), and again averaging over one wave period,

$$
P \equiv \frac{1}{2 \pi} \int_{0}^{2 \pi} \mathrm{d} \alpha \rho=\frac{1}{2}\left[\mathrm{~K}^{2} \mathrm{~A} \cos (\alpha)\right]^{2},
$$




\section{The Kinematic Theory of Wavetrains}

Here we derive the fundamental properties of wavetrains, or almost purely sinusoidal waveforms, in the presence of constant or slowly changing mean winds. The material presented in this section is condensed from Lighthill (1978), and is necessary to the derivation of the conservation laws in the following section. The kinematic theory demonstrates the key role played by the group velocity, and is the basis of ray tracing, a technique which has recently found widespread application (Karoly and Hoskins, 1982).

\section{3a. Constant Mean Wind}

We let the mean wind have components in both horizontal directions, so that both $\bar{u}$ and $\bar{v}$ are non-zero, although they are both constant. In this case $(2.7)$ reduces to

$$
\frac{\partial q^{\prime}}{\partial t}+\bar{u} \frac{\partial q^{\prime}}{\partial x}+\bar{v} \frac{\partial q^{\prime}}{\partial y}+B v^{\prime}=0
$$

for which $(2.10)$ is a valid solution, with

$$
\omega=(\bar{u} k+\bar{v} \ell)-\frac{\beta k}{k^{2}} .
$$

Here we do not need the specific form of the dispersion relation, we only need the fact that there is one, i.e.

$$
\omega=\Omega(k, l, \mathrm{~m}) .
$$

In the presence of the constant mean wind $(\bar{u}, \bar{v})$, a more general solution of the linearized equations can be constructed as a superposition of solutions of the form of (2.10). The approach adopted here, however, is somewhat different. We consider a solution which locally is given by (2.10), but in which the amplitude (A), frequency $(\omega)$ and vector wavenumber $(k, l, m)$ change slowly in space and time. Physically, this corresponds to the assumption that one has waited long enough after an initial disturbance for the different components of the spectrum (waves 
of different wavenumbers) to be separated in space from each other, i.e. for the disturbance to have dispersed. The amplitude, frequency and wavenumber components change by a small fraction of themselves over the local wavelength or local period.

We start with the generalized form of (2.10)

$$
\psi^{\prime}=A(x ; y, z, t) e^{z /(2 H)} \cos (\alpha(x, y, z, t))
$$

and expand the total phase $\alpha$ in deviations ( $\delta x, \delta y, \delta z, \delta t)$ about some point $\left(x_{0}, y_{0}, z_{0}, t_{0}\right):$

$$
\begin{aligned}
\alpha(x, y, z, t)=\alpha\left(x_{0}, y_{0}, z_{0}, t_{0}\right) & +\frac{\partial \alpha}{\partial t} \delta t+\frac{\partial \alpha}{\partial x} \delta x+\frac{\partial \alpha}{\partial y} \delta y+\frac{\partial \alpha}{\partial z} \delta z \\
& +o\left((\delta t)^{2},(\delta x)^{2}, \ldots\right)+\ldots
\end{aligned}
$$

We can, without loss of generality, set $\alpha\left(x_{0}, y_{0}, z_{0}, t_{0}\right)=0$, and, neglecting second order terms :

$$
\alpha(x, y, z, t)=\frac{\partial \alpha}{\partial t} \delta t+\frac{\partial \alpha}{\partial x} \delta x+\frac{\partial \alpha}{\partial y} \delta y+\frac{\partial \alpha}{\partial z} \delta_{z}
$$

Since $\alpha$ locally takes the form

$$
\alpha=k x+\ell y+m z-\omega t,
$$

the local frequency $w$ and wavenumber vector $(k, \ell, m)$ are defined as:

$$
\begin{aligned}
\omega & =-\frac{\partial \alpha}{\partial t} \\
(k, \ell, m) & =\left(\frac{\partial \alpha}{\partial x}, \frac{\partial \alpha}{\partial y}, \frac{\partial \alpha}{\partial z}\right) .
\end{aligned}
$$

We now come to one of the most important ingredients in the theory, the step at which the physics of the wave enters the formalism. It is assumed that the dispersion relation (3.1) holds for all space and time, with the frequency and wavenumber being given by their local values. From (3.1) and (3.5a), (3.5b), this gives: 


$$
-\frac{\partial \alpha}{\partial t}=\Omega\left(\frac{\partial \alpha}{\partial x}, \frac{\partial \alpha}{\partial y}, \frac{\partial \alpha}{\partial z}\right)
$$

Differentiating (3.6) with respect to $x, y$ and $z$, separately, gives:

$$
\begin{aligned}
& -\frac{\partial^{2} \alpha}{\partial \mathrm{x} \partial \mathrm{t}}=\frac{\partial \Omega}{\partial \mathrm{k}}\left(\frac{\partial^{2} \alpha}{\partial \mathrm{x}^{2}}\right)+\frac{\partial \Omega}{\partial \ell}\left(\frac{\partial^{2} \alpha}{\partial \mathrm{x} \partial \mathrm{y}}\right)+\frac{\partial \Omega}{\partial \mathrm{m}}\left(\frac{\partial^{2} \alpha}{\partial \mathrm{x} \partial \mathrm{z}}\right), \\
& -\frac{\partial^{2} \alpha}{\partial \mathrm{y} \partial \mathrm{t}}=\frac{\partial \Omega}{\partial \mathrm{k}}\left(\frac{\partial^{2} \alpha}{\partial \mathrm{x} \partial \mathrm{y}}\right)+\frac{\partial \Omega}{\partial \ell}\left(\frac{\partial^{2} \alpha}{\partial \mathrm{y}^{2}}\right)+\frac{\partial \Omega}{\partial \mathrm{m}}\left(\frac{\partial^{2} \alpha}{\partial \mathrm{y} \partial}\right), \\
& -\frac{\partial^{2} \alpha}{\partial \mathrm{z} \partial t}=\frac{\partial \Omega}{\partial \mathrm{k}}\left(\frac{\partial^{2} \alpha}{\partial \mathrm{x} \partial \mathrm{z}}\right)+\frac{\partial \Omega}{\partial \ell}\left(\frac{\partial^{2} \alpha}{\partial \mathrm{y} \partial \mathrm{z}}\right)+\frac{\partial \Omega}{\partial \mathrm{m}}\left(\frac{\partial^{2} \alpha}{\partial \mathrm{z}^{2}}\right) .
\end{aligned}
$$

If we define the group velocity $\vec{C}$ as:

$$
\vec{c}=\left(c_{x}, c_{y}, c_{z}\right)=\left(\frac{\partial \Omega}{\partial_{k}}, \frac{\partial \Omega}{\partial \ell}, \frac{\partial \Omega}{\partial_{m}}\right),
$$

and use (3.5a) and (3.5b) to derive relationships of the type

$$
\frac{\partial^{2} \alpha}{\partial x \partial t}=-\frac{\partial \omega}{\partial x}=+\frac{\partial k}{\partial t} ; \frac{\partial \alpha^{2}}{\partial x^{2}}=\frac{\partial k}{\partial x} ; \text { etc. }
$$

equations $(3.7 a)-(3.7 c)$ become

$$
\begin{gathered}
\frac{\partial \mathrm{k}}{\partial t}+\vec{C} \cdot \overrightarrow{\nabla_{3}} \mathrm{k}=0 \\
\frac{\partial \ell}{\partial t}+\vec{C} \cdot \overrightarrow{\nabla_{3}} \ell=0 \\
\overrightarrow{\partial m}+\vec{C} \cdot \overrightarrow{\nabla_{3} m}=0 .
\end{gathered}
$$

$\rightarrow$ 
here and throughout this article, (3.10a) - (3.10c) are just

$$
\begin{aligned}
& \frac{D k}{D t}=0 \\
& \frac{D \ell}{D t}=0 \\
& \frac{D m}{D t}=0
\end{aligned}
$$

Further, $\omega$ is also constant following the group velocity, for

$$
\frac{\mathrm{D} \omega}{\mathrm{D} t}=\frac{\partial \Omega}{\partial \mathrm{k}} \frac{\mathrm{Dk}}{\mathrm{Dt}}+\frac{\partial \Omega}{\partial \ell} \frac{\mathrm{D}}{\mathrm{D} t}+\frac{\partial \Omega}{\partial \mathrm{m}} \frac{\mathrm{Dm}}{\mathrm{Dt}}=0
$$

3b. Spatially varying mean wind.

We are now in a position to introduce slow spatial variations in the mean wind, slow in the sense that, as before, there is a small fractional change over a wavelength. We assume the mean wind to be constant in time. The dispersion relation is still assumed to hold for all space and time, but since the dispersion relation generally involves the mean wind, which is no longer constant, (3.1) must be revised. The frequency w now has a (slow) dependence on the coordinates beyond that given by the (slow) coordinate dependence of $(k, \ell, m)$. This additional dependence of $\omega$ on $(x, y, z)$ is formally introduced by letting $\omega$ depend on the parmaters $\lambda_{i}, i=1, \ldots \ldots, N$. (3.1) then becomes

$$
\omega=\Omega\left(k, \ell, m ; \lambda_{i}\right) \text {. }
$$

For most applications, the $\lambda_{i}$ can be taken to be the components of the mean wind: $\lambda_{1}=\bar{u}, \lambda_{2}=\bar{v}$. Equation (3.6) becomes

$$
-\frac{\partial \alpha}{\partial t}=\Omega\left(\frac{\partial \alpha}{\partial x}, \frac{\partial \alpha}{\partial y} ; \frac{\partial \alpha}{\partial z} ; \lambda_{i}\right),
$$

and $(3.7 a)-(3.7 c)$ acquire the additional terms 


$$
\begin{aligned}
& -\frac{\partial^{2} \alpha}{\partial x \partial t}=\ldots \ldots+\sum_{i} \frac{\partial \Omega}{\partial \lambda_{i}} \frac{\partial \lambda_{i}}{\partial x} \\
& -\frac{\partial^{2} \alpha}{\partial y \partial t}=\ldots \ldots+\sum_{i} \cdot \frac{\partial \Omega}{\partial \lambda_{i}} \frac{\partial \lambda_{i}}{\partial y} \\
& -\frac{\partial^{2} \alpha}{\partial z \partial t}=\ldots+\sum_{i} \frac{\partial \Omega}{\partial \lambda_{i}} \frac{\partial \lambda_{i}}{\partial z},
\end{aligned}
$$

while $(3.12 a)-(3.12 c)$ become

$$
\begin{aligned}
& \frac{\mathrm{Dk}}{\mathrm{Dt}}=-\sum_{i} \frac{\partial \Omega}{\partial \lambda_{i}} \frac{\partial \lambda_{i}}{\partial \mathrm{x}} \\
& \frac{\mathrm{Dl}}{\mathrm{Dt}}=-\sum_{i} \frac{\partial \Omega}{\partial \lambda_{i}} \frac{\partial \lambda_{i}}{\partial \mathrm{y}} \\
& \frac{\mathrm{Dm}}{\mathrm{Dt}}=-\sum_{i} \frac{\partial \Omega}{\partial \lambda_{i}} \cdot \frac{\partial \lambda_{i}}{\partial z}
\end{aligned}
$$

Finally $(3.13)$ becomes

$$
\frac{D \omega}{D t}=\frac{\partial \Omega}{\partial k} \frac{D k}{D t}+\frac{\partial \Omega}{\partial l} \frac{D l}{D t}+\frac{\partial \Omega}{\partial m} \frac{D m}{D t}+\sum_{i} \frac{\partial \Omega}{\partial \lambda_{i}} \frac{D \lambda_{i}}{D t} .
$$

From $(3.17 a)-(3.17 c)$ and $(3.18)$, we prove a remarkable result:

$$
\begin{aligned}
& \frac{D \omega}{D t}=-\frac{\partial \Omega}{\partial k}\left[\sum_{i} \frac{\partial \Omega}{\partial \lambda_{i}} \frac{\partial \lambda_{i}}{\partial x}\right]-\frac{\partial \Omega}{\partial \ell}\left[\sum_{i} \frac{\partial \Omega}{\partial \lambda_{i}} \frac{\partial}{\partial y} \lambda_{i}\right]-\frac{\partial \Omega}{\partial m}\left[\sum_{i} \frac{\partial \Omega}{\partial} \frac{\partial}{\lambda_{i}} \frac{\partial \lambda_{i}}{\partial z}\right] \\
& +\sum_{i} \frac{\partial \Omega}{\partial \lambda_{i}}\left[\frac{\partial \bar{\lambda}_{i}}{\partial t}+c_{x} \frac{\partial \bar{\lambda}_{i}}{\partial x}+c_{y} \frac{\partial \bar{\lambda}_{i}}{\partial y}+c_{z} \frac{\partial \lambda_{i}}{\partial z}\right]
\end{aligned}
$$

where we have used (3.11). Noting that $\frac{\partial \lambda_{i}}{\partial t}=0$ (mean winds constant in time), and using ( 3.8$)$, we easily find that

$$
\frac{\mathrm{D} \omega}{\mathrm{Dt}}=0
$$


The most important source of dependence of $\Omega$ on the $\lambda_{i}$ arises from the advective effect of the mean flow. This dependence can be expressed by introducing the transformation to the frame of reference moving with the local mean flow, i.e. the frame of reference in which the local mean flow vanishes. The coordinates in the transformed frame are denoted by primes. We have

$$
\begin{aligned}
& x^{\prime}=x-\bar{u} t \\
& y^{\prime}=y-\bar{v} t \\
& z^{\prime}=z \\
& t^{\prime}=t
\end{aligned}
$$

We obtain the transformed wavenumber $\left(k^{-}, \ell^{-}, m^{\prime}\right)$ and frequency $\left(\omega^{-}\right)$by noting that the local phase $\alpha$ is invariant, so that to first order in the derivatives of $\alpha$,

$$
\left(-\omega^{\prime} t^{\prime}+k^{\prime} x^{\prime}+\ell^{\prime} y^{\prime}+m^{\prime} z^{\prime}\right)=(-\omega t+k x+l y+m z)
$$

for all (x, y, $z, t)$. From (3.20), this becomes

$$
\begin{aligned}
{\left[-\left(\omega^{\prime}+k^{-} \bar{u}+\ell^{-} \vec{v}\right) t\right.} & \left.+k^{\prime} x+\ell^{\prime} y+m^{\prime} z\right] \\
& =[-\omega t+k x+\ell y+m z]
\end{aligned}
$$

whence

$$
\begin{aligned}
& \mathrm{k}=\mathrm{k}^{\prime}, \ell=\ell^{\prime}, \mathrm{m}=\mathrm{m}^{\prime}, \text { and } \\
& \omega=\omega^{\prime}+\mathrm{k} \overline{\mathrm{u}}+\ell \overline{\mathrm{v}}
\end{aligned}
$$

$\omega^{-}$is called the intrinsic frequency.

We may not have transformed away all the dependence on the variable mean wind, however, for it is possible that $\omega^{\prime}$ still depends on $\bar{u}, \bar{v}$. An example of this arises in the study of Rossby waves in shallow water theory (Pedlosky, 1979). In that problem $\omega^{\prime}$ depends on $\bar{u}$ via a term arising from the perturbation advection of mean state potential vorticity (Pedlosky, pp.91, 109). Physically, the mean current $\bar{u}$ is balanced by a slope in the $y$ direction of the free surface, a slope 
which is the same in any frame of reference moving in the $x$ direction. In the case treated in the previous and subsequent sections, namely Rossby waves in a stratified fluid, $\omega^{\prime}$ is independent of $(\bar{u}, \bar{v})$, and depends on $(x, y, z, t)$ only through $(k, \ell, m)$.

Analogously to (3.14), we can write

$$
\omega^{-}=\Omega^{-}\left(k, \ell, m ; \lambda_{i}\right),
$$

where $\lambda_{i}$ again refers to $\bar{u}$ and $\bar{v}$ for $i=1$ and 2 .

From (3.23) and (3.24),

$$
\omega=k \bar{u}+\ell \bar{v}+\Omega^{-}\left(k, \ell, m ; \lambda_{i}\right)
$$

The right hand sides of $(3.16 a)-(3.16 \mathrm{c})$ can now be written as:

$$
\begin{aligned}
& \sum_{i} \frac{\partial \Omega}{\partial \lambda_{i}} \frac{\partial \lambda_{i}}{\partial x}=k \frac{\partial \bar{u}}{\partial x}+\ell \frac{\partial \bar{v}}{\partial x}+\sum_{i} \frac{\partial \Omega^{-}}{\partial \lambda_{i}} \frac{\partial \lambda_{i}}{\partial x}, \\
& \sum_{i} \frac{\partial \Omega}{\partial \lambda_{i}} \frac{\partial \lambda_{i}}{\partial y}=k \frac{\partial \bar{u}}{\partial y}+\ell \frac{\partial \bar{v}}{\partial y}+\sum_{i} \frac{\partial \Omega^{\prime}}{\partial \lambda_{i}} \frac{\partial \lambda_{i}}{\partial y}, \\
& \sum_{i} \frac{\partial \Omega}{\partial \lambda_{i}} \frac{\partial \lambda_{i}}{\partial z}=k \frac{\partial \bar{u}}{\partial z}+\ell \frac{\partial \bar{v}}{\partial z}+\sum_{i} \frac{\partial \Omega^{\prime}}{\partial \lambda_{i}} \cdot \frac{\partial \lambda_{i}}{\partial z} .
\end{aligned}
$$

Here $(k, l, m)$ have not been differentiated, since it is only the coordinate dependence beyond the dependence through $(k, \ell, m)$ that is being considered. Finally, combining $(3.26 a)-(3.26 c)$ with $(3.17 a)-(3.17 c)$ and specializing to the case of Rossby waves in a stratified fluid $\left(\frac{\partial \Omega^{-}}{\partial \lambda_{i}}=0\right)$, we have

$$
\begin{aligned}
& \frac{D k}{D t}=-k \frac{\partial \bar{u}}{\partial x}-\ell \frac{\partial \bar{v}}{\partial x} \\
& \frac{D \ell}{D t}=-k \frac{\partial \bar{u}}{\partial y}-\ell \frac{\partial \bar{v}}{\partial y} \\
& \frac{D m}{D t}=-k \frac{\partial \bar{u}}{\partial z}-\ell \frac{\partial \bar{v}}{\partial z}
\end{aligned}
$$


4. Conservation Laws for Wave Energy, Wave Action and Wave Enstrophy in a Slowly Varying Mean Flow

4a. Conservation law for wave energy

We start with a full linearized equation (2.7), in which $\bar{u}$ and $\bar{v}$ may vary slowly in space:

$$
\frac{\partial q^{\prime}}{\partial t}+\bar{u} \frac{\partial q^{\prime}}{\partial x}+\bar{v} \frac{\partial q^{\prime}}{\partial y}+u^{\prime} \frac{\partial \bar{q}}{\partial x}+v^{\prime} \frac{\partial \bar{q}}{\partial y}=0
$$

where $\bar{q}$ is given by $(2.6 a)$. With $\bar{u}=-\frac{\partial \bar{\psi}}{\partial y}, \bar{v}=\frac{\partial \bar{\psi}}{\partial x}$,

$$
\frac{\partial \bar{q}}{\partial x}=\nabla_{2}^{2} \bar{v}+\frac{1}{S} e^{z / H} \frac{\partial}{\partial z}\left(e^{-z / H} \frac{\partial \vec{v}}{\partial z}\right)
$$

and

$$
\frac{\partial \bar{q}}{\partial y}=\beta+\nabla_{2}^{2} \bar{u}+\frac{1}{\text { S }} e^{z / H} \frac{\partial}{\partial z}\left(e^{-z / H} \frac{\partial \bar{u}}{\partial z}\right)
$$

Since the mean flow is assumed to vary slowly in space, we neglect second derivatives of $(\bar{u}, \bar{v})$ in $(4.1)$.

Thus,

$$
\left.\begin{array}{l}
\frac{\partial \bar{q}}{\partial x}=0, \\
\frac{\partial \bar{q}}{\partial y} \simeq B,
\end{array}\right\}
$$

and $(4.1)$ becomes

$$
\left(\frac{\partial}{\partial t}+\bar{u} \frac{\partial}{\partial x}+\bar{v} \frac{\partial}{\partial y}\right) q^{\prime}+\beta v^{\prime}=0,
$$

where $q^{\prime}$ is given by $(2.6 b)$. We try a solution of the form $(2.10)$, but in which the amplitude $A$ and vector wavenumber $(k, \ell, m)$ are allowed to vary slowly in space and time. The variations of $(k, \ell, m)$ obey the relations derived in Section 3 , especially equations (3.9) and (3.27). The frequency $w$ 
is given by the local dispersion relationship, while the variation of the amplitude $A$ is discussed is the following paragraphs.

Our basic solution is

$$
\psi^{\prime}=A(x, y, z, t) e^{z /(2 H)} \cos (k x+\ell y+m z-\omega t) \equiv A(x, y, z, t) e^{z /(2 H)} \cos (\alpha) \cdot(4 \cdot 5)
$$

We substitute (4.5) into (4.4), using (2.6b). A number of intermediate results are helpful:

$$
\begin{aligned}
& \frac{\partial \psi^{-}}{\partial x}=\frac{\partial A}{\partial x} e^{z /(2 H)} \cos (\alpha)-k A e^{z /(2 H)} \sin (\alpha) \\
& \frac{\partial^{2} \psi^{-}}{\partial x^{2}}=\frac{\partial^{2} A}{\partial x^{2}} e^{z /(2 H)} \cos (\alpha)-2 k \cdot \frac{\partial A}{\partial x} e^{z /(2 H)} \sin (\alpha) \\
& -\frac{\partial k}{\partial x} \mathrm{~A} \mathrm{e}^{z /(2 \mathrm{H})} \sin (\alpha)-\mathrm{k}^{2} \mathrm{~A} \mathrm{e}^{\mathrm{z} /(2 \mathrm{H})} \cos (\alpha)
\end{aligned}
$$

A result analogous to $(4.6 \mathrm{~b})$ holds for $\frac{\partial^{2} \psi^{-}}{\partial y^{2}}$, so that

$$
\begin{aligned}
& \nabla_{2}^{2} \psi^{-}=\underbrace{\nabla_{2}^{2} \mathrm{~A} \mathrm{e}} \underbrace{z /(2 \mathrm{H})} \cos (\alpha)-2\left(\mathrm{k} \frac{\partial \mathrm{A}}{\partial \mathrm{x}}+\ell \frac{\partial \mathrm{A}}{\partial \mathrm{y}}\right) \mathrm{e}^{\mathrm{z} /(2 \mathrm{H})} \sin (\alpha) \\
& \text { - A }\left(\frac{\partial k}{\partial x}+\frac{\partial \ell}{\partial y}\right) e^{z /(2 H)} \sin (\alpha)-\left(k^{2}+\ell^{2}\right) A e^{z /(2 H)} \cos (\alpha) \text {. }
\end{aligned}
$$


Also,

$\frac{1}{\widehat{S}} e^{z / H} \frac{\partial}{\partial z}\left(e^{-z / H} \frac{\partial \psi^{\prime}}{\partial z}\right)=\frac{1}{\widehat{S}} e^{z / H} \frac{\partial}{\partial z}\left[e^{-z / H}\left(\frac{\partial A}{\partial z} \cos (\alpha)\right.\right.$

$$
\left.\left.+\frac{A}{2 H} \cos (\alpha)-\operatorname{mAsin}(\alpha)\right)\right]
$$

$=\frac{1}{\widehat{S}} e^{z / H}\left[-\frac{1}{2 H} e^{-z /(2 H)}\left(\frac{\partial A}{\partial z} \cos (\alpha)+\frac{A}{2 H} \cos (\alpha)-A \sin (\alpha)\right)\right.$

$+e^{-z /(2 H)}\left\{\frac{\partial^{2} A}{\partial z^{2}} \cos (\alpha)-2 m \frac{\partial A}{\partial z} \sin (\alpha)+\frac{1}{2 H} \frac{\partial A}{\partial z} \cos (\alpha)-\frac{m A}{2 H} \sin (\alpha)\right.$ $\left.\left.-\frac{\partial m}{\partial z} A \sin (\alpha)-m^{2} A \cos (\alpha) \quad\right\}\right]$

$=\frac{1}{\widehat{S}} e^{z /(2 H)}\left[\cos (\alpha) \cdot\left\{\frac{\partial^{2} A}{\partial z^{2}}-\frac{1}{2 H} \frac{\partial A}{\partial z}-\frac{A}{4 H^{2}}+\frac{1}{2 H} \frac{\partial A}{\partial z}-m^{2} A\right\}\right.$

$\left.+\sin (\alpha) \cdot\left\{\frac{m A}{2 H}-2 m \frac{\partial A}{\partial z}-\frac{m A}{2 H}-A \frac{\partial m}{\partial z}\right\}\right]$

$=\frac{1}{\widehat{S}} e^{z /(2 H)}\left[\cos (\alpha)\left\{\frac{\partial^{2} A}{\partial z^{2}}-A\left(m^{2}+\frac{1}{4 H^{2}}\right)\right\}+\sin (\alpha)\left\{-2 m \frac{\partial A}{\partial z}-A \frac{\partial m}{\partial z}\right\}\right]_{(4.6 d)}$

Further,

$$
\left.\begin{array}{l}
\left(\frac{\partial}{\partial t}+\bar{u} \frac{\partial}{\partial x}+\bar{v} \frac{\partial}{\partial y}\right) \cos (\alpha)=(\omega-k \vec{u}-\ell \bar{v}) \sin (\alpha) \\
\left(\frac{\partial}{\partial t}+\bar{u} \frac{\partial}{\partial x}+\bar{v} \frac{\partial}{\partial y}\right) \sin (\alpha)=-(\omega-k \bar{u}-\overline{l v}) \cos (\alpha)
\end{array}\right\}
$$

Since the amplitude is assumed to vary slowly, we will neglect the underlined terms in $(4.6 \mathrm{~b}),(4.6 \mathrm{c})$ and $(4.6 \mathrm{~d})$, all of which involve second spatial derivatives of $A$. For example, the neglect of $\frac{\partial^{2} A}{\partial x^{2}}$ in (4.6b) compared to $2 k \frac{\partial A}{\partial x}$ is equivalent to the statement that $\frac{\partial^{2} A}{\partial x^{2}} \Lambda \ll \frac{\partial A}{\partial x}$, where $\Lambda$ is the wavelength in the $x$ direction; which is just the statement that the change of $\frac{\partial A}{\partial x}$ over one wavelength is small. 


$$
\begin{aligned}
& \text { From }(4.6 \mathrm{a})-(4.6 \mathrm{e}) \text {, and }(2.6 \mathrm{~b}), \\
& \mathrm{q}^{\prime}=\mathrm{e}^{z /(2 \mathrm{H})}\left[\cos (\alpha) \cdot\left\{-\mathrm{A}\left(\mathrm{k}^{2}+\ell^{2}+\frac{1}{\widehat{S}}\left(\mathrm{~m}^{2}+\frac{1}{4 \mathrm{H}^{2}}\right)\right)\right\}\right. \\
& \left.+\sin (\alpha)\left\{-\mathrm{A}\left(\frac{\partial \mathrm{k}}{\partial \mathrm{x}}+\frac{\partial \ell}{\partial y}+\frac{1}{\widehat{S}} \frac{\partial \mathrm{m}}{\partial z}\right)-2\left(\mathrm{k} \frac{\partial \mathrm{A}}{\partial \mathrm{x}}+\ell \frac{\partial A}{\partial y}+\frac{1}{\widehat{S}} \mathrm{~m} \frac{\partial A}{\partial z}\right)\right\}\right] .
\end{aligned}
$$

Finally (4.4) becomes

$$
\begin{aligned}
& (\omega-\mathrm{k} \overline{\mathrm{u}}-\ell \bar{v}) \cdot \sin (\alpha) \cdot\left(-\mathrm{AK}^{2}\right)+(\omega-\mathrm{k} \overline{\mathrm{u}}-\overline{\ell \mathrm{v}}) \cdot \cos (\alpha) \cdot\left[\mathrm{A}\left(\frac{\partial \mathrm{k}}{\partial \mathrm{x}}+\frac{\partial \ell}{\partial \mathrm{y}}+\frac{1}{\mathrm{~S}} \frac{\partial \mathrm{m}}{\partial z}\right)\right. \\
& \left.\quad+2\left(\mathrm{k} \frac{\partial \mathrm{A}}{\partial \mathrm{x}}+\ell \frac{\partial \mathrm{A}}{\partial \mathrm{y}}+\frac{1}{\widehat{\mathrm{s}}} \mathrm{m} \frac{\partial \mathrm{A}}{\partial z}\right)\right]
\end{aligned}
$$

$+\cos (\alpha)\left[-\mathrm{K}^{2} \frac{\mathrm{dA}}{\mathrm{dt}}-2 \mathrm{~A}\left(\mathrm{k} \frac{\mathrm{dk}}{\mathrm{dt}}+\ell \frac{\mathrm{d} \ell}{\mathrm{dt}}+\frac{1}{\mathrm{~S}} \mathrm{~m} \frac{\mathrm{dm}}{\mathrm{dt}}\right)\right]$

$+\sin (\alpha)\left[-\frac{d A}{d t}\left(\frac{\partial k}{\partial x}+\frac{\partial \ell}{\partial y}+\frac{1}{\widehat{S}} \frac{\partial m}{\partial z}\right)-A \frac{d}{d t}\left(\frac{\partial k}{\partial x}+\frac{\partial \ell}{\partial y}+\frac{1}{\widehat{S}} \frac{\partial m}{\partial z}\right)\right.$

$-2\left(\frac{d k}{d t} \frac{\partial A}{\partial x}+\frac{d \ell}{d t} \frac{\partial A}{\partial y}+\frac{1}{S} \frac{d m}{d t} \frac{\partial A}{\partial z}\right)-2\left(k \frac{d}{d t} \frac{\partial A}{\partial x}+\ell \frac{d}{d t} \frac{\partial A}{\partial y}\right.$

$\left.\left.+\frac{1}{\hat{S}} m \frac{d}{d t} \frac{\partial A}{\partial z}\right)\right] *+\beta \frac{\partial A}{\partial x} \cos (\alpha)-\beta k A \sin (\alpha)=0$.

In $(4.7), \mathrm{K}^{2}$ is given by $(2.12)$,

$$
\mathrm{K}^{2}=\mathrm{k}^{2}+\ell^{2}+\frac{1}{\mathrm{~S}}\left(\mathrm{~m}^{2}+\frac{1}{4 \mathrm{H}^{2}}\right),
$$

and $\frac{d}{d t}$ is defined by

$$
\frac{d}{d t} \equiv \frac{\partial}{\partial t}+\bar{u} \frac{\partial}{\partial x}+\bar{v} \frac{\partial}{\partial y}
$$

Both the operator $\frac{d}{d t}$ defined above and the operator $\frac{D}{D t}$ defined in (3.11) will be needed in the subsequent development. The term with the asterisk in (4.7) can be neglected, since it contains second derivatives of the slowly varying quantities 
$\mathrm{A},(\mathrm{k}, \ell, \mathrm{m})$ or products of their first derivatives. (4.7) is really two equations, one for the coefficients of the $\sin (\alpha)$ term, the other for the coefficients of the $\cos (\alpha)$ term. Setting the sum of all coefficients of $\sin (\alpha)$ to zero, we get

$$
-(w-k \bar{u}-\ell \bar{v}) A K^{2}-B k A=0
$$

or

$$
w=k \bar{u}+\ell \bar{v}-\frac{B k}{K^{2}}
$$

which is just the generalization of (2.13) to the case of non-zero mean flow in the $y$ direction.

The equation obtained by setting the sum of the coefficients of $\cos (\alpha)$ to zero in $(4.7)$ is :

$$
\begin{aligned}
& (w-\hat{k} \bar{u}-\overline{\ell v})\left[A\left(\frac{\partial k}{\partial x}+\frac{\partial \ell}{\partial y}+\frac{1}{\widehat{S}} \frac{\partial m}{\partial z}\right)+2\left(k \frac{\partial A}{\partial x}+\ell \frac{\partial A}{\partial y}+\frac{1}{\widehat{S}} m \frac{\partial A}{\partial z}\right)\right] \\
& -K^{2} \frac{d A}{d t}-A \frac{d}{d t} K^{2}+B \frac{\partial A}{\partial x}=0 .
\end{aligned}
$$

With the help of $(4.9 \mathrm{~b}),(4.10)$ becomes

$$
\begin{gathered}
\frac{-\beta k}{k^{2}}\left[A\left(\frac{\partial k}{\partial x}+\frac{\partial \ell}{\partial y}+\frac{1}{S} \frac{\partial m}{\partial z}\right)+2\left(k \frac{\partial A}{\partial x}+\ell \frac{\partial A}{\partial y}+\frac{1}{S} m \frac{\partial A}{\partial z}\right)\right] \\
-K^{2} \frac{d A}{d t}-A \frac{d}{d t} K^{2}+B \frac{\partial A}{\partial x}=0 .
\end{gathered}
$$

or

$$
\frac{\mathrm{dA}}{\mathrm{dt}}+\frac{2 \beta \mathrm{k}}{\mathrm{K}^{4}}\left(\mathrm{k} \frac{\partial \mathrm{A}}{\partial \mathrm{x}}+\ell \frac{\partial \mathrm{A}}{\partial \mathrm{y}}+\frac{1}{\hat{\mathrm{S}}} \mathrm{m} \frac{\partial \mathrm{A}}{\partial z}\right)-\frac{B}{\mathrm{k}^{2}}-\frac{\partial \mathrm{A}}{\partial \mathrm{x}}
$$

$$
=\frac{-A}{k^{2}} \frac{d}{d t} k^{2}-B \frac{k A}{K^{4}}\left[\frac{\partial k}{\partial x}+\frac{\partial \ell}{\partial y}+\frac{1}{\hat{S}} \frac{\partial m}{\partial z}\right]
$$

To make further progress, we must calculate the group velocity $C$ where, as in (3.8), 


$$
\vec{C}=\left(C_{x}, C_{y}, C_{x}\right)=\left(\frac{\partial \omega}{\partial k}, \frac{\partial \omega}{\partial \ell}, \frac{\partial \omega}{\partial m}\right) .
$$

We have, from $(\dot{4} .9 \mathrm{~b})$

$$
\begin{aligned}
& C_{x}=\frac{\partial \omega}{\partial k}=\bar{u}-\frac{\beta}{k^{2}}+\frac{2 \beta k^{2}}{k^{4}} \equiv \bar{u}+C_{x}{ }^{\prime}, \\
& C_{y}=\frac{\partial \omega}{\partial \ell}=\bar{v}+\frac{2 B k \ell}{k^{4}} \equiv \bar{v}+C_{y}^{\prime}, \\
& C_{z}=\frac{\partial \omega}{\partial m}=+\frac{2 B k m}{\widehat{S k^{4}}} \equiv C_{z}{ }^{\prime},
\end{aligned}
$$

Equations (4.12) define the intrinsic group velocity $\vec{c} \vec{c}$, but it is also given by $\vec{c}^{-}=\left(\frac{\partial \omega^{\prime}}{\partial k}, \frac{\partial \omega^{\prime}}{\partial l}, \frac{\partial \omega^{\prime}}{\partial m}\right)$, where $\omega^{-}=\omega-k \bar{u}-\ell \bar{v}$ is the intrinsic frequency as defined in (3.23). Using $(4.12 a)-(4.12 c)$ in $(4.11 b)$, we get:

$$
\begin{aligned}
\frac{\partial A}{\partial t}+C_{x} \frac{\partial A}{\partial x} & +C_{y} \frac{\partial A}{\partial y}+C_{z} \frac{\partial A}{\partial z} \\
& =\frac{D A}{D t}=-\frac{A}{K^{2}} \frac{d}{d t}\left(K^{2}\right)-\frac{\beta k A}{K^{4}}\left[\frac{\partial k}{\partial x}+\frac{\partial l}{\partial y}+\frac{1}{S} \frac{\partial m}{\partial z}\right] .
\end{aligned}
$$

Now, from $(2.16)$,

$$
\frac{D E}{D t}=\frac{1}{2} A K^{2} \frac{D A}{D t}+\frac{1}{4} A^{2} \frac{D}{D t}\left(K^{2}\right)
$$

Multiplying (4.13) by $\frac{1}{2} A K^{2}$, and adding $\frac{1}{4} A^{2}-\frac{D}{D t}\left(K^{2}\right)$ to both sides, we get:

$$
\frac{D E}{D t}=\frac{1}{4} A^{2} \frac{D}{D t}\left(K^{2}\right)-\frac{1}{2} A^{2} \frac{d}{d t}\left(K^{2}\right)-\frac{1}{2} \frac{\beta k A^{2}}{K^{2}}\left[\frac{\partial k}{\partial x}+\frac{\partial \ell}{\partial y}+\frac{1}{\hat{S}} \frac{\partial m}{\partial z}\right],
$$

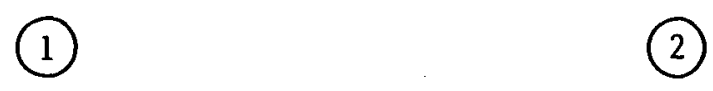

where it must be kept in mind that the operators $\frac{d}{d t}$ and $\frac{D}{D t}$ are not identical. 
Term 1 in (4.15) can be further developed:

$$
\begin{aligned}
& \text { (1) }=\frac{1}{4} A^{2} \frac{D}{D t}\left(k^{2}\right)-\frac{1}{2} A^{2} \frac{D}{D t}\left(K^{2}\right)+\frac{1}{2} A^{2}\left(C_{x}^{-} \frac{\partial}{\partial x}+C_{y}^{-} \frac{\partial}{\partial y}+C_{z} \cdot \frac{\partial}{\partial z}\right) K^{2} \\
& =-\frac{1}{4} A^{2} \frac{D}{D t}\left(K^{2}\right)+\frac{1}{2} A^{2}\left[\left(\frac{-\beta}{K^{2}}+\frac{2 \beta k^{2}}{K^{4}}\right) \frac{\partial}{\partial x} K^{2}+\frac{2 \beta k \ell}{K^{4}} \frac{\partial}{\partial y} K^{2}\right. \\
& \left.+\frac{2 \mathrm{~km}}{\widehat{S} \mathrm{~K}^{4}} \frac{\partial}{\partial z} \mathrm{~K}^{2}\right] \text {, }
\end{aligned}
$$

so that

$$
\begin{aligned}
(1)=-\frac{1}{2} A^{2}\left[k \frac{D k}{D t}\right. & \left.+\ell \frac{D \ell}{D t}+\frac{1}{\widehat{S}} m \frac{D m}{D t}\right]+\frac{1}{2} A^{2}\left[\left(\frac{-\beta}{\mathrm{K}^{2}}+\frac{2 \beta k^{2}}{\mathrm{~K}^{4}}\right) \frac{\partial}{\partial \mathrm{x}} \mathrm{K}^{2}\right. \\
& \left.+\frac{2 B k \ell}{\mathrm{K}^{4}} \frac{\partial}{\partial \mathrm{y}} \mathrm{K}^{2}+\frac{2 B \mathrm{~km}}{\widehat{S} \mathrm{~K}^{4}} \frac{\partial}{\partial z} \mathrm{~K}^{2}\right] .
\end{aligned}
$$

Using (3.27a) $-(3.27 c)$ in $(4.16)$, and putting the result back into (4.15) gives us:

$$
\begin{aligned}
\frac{D E}{D t} & =\frac{1}{2} A^{2}\left[k\left(k \frac{\partial \vec{u}}{\partial x}+\ell \frac{\partial \bar{v}}{\partial x}\right)+\ell\left(k \frac{\partial \bar{u}}{\partial y}+\ell \frac{\partial \bar{v}}{\partial y}\right)+\frac{1}{\widehat{s}} m\left(k \frac{\partial \bar{u}}{\partial z}+\ell \frac{\partial \bar{v}}{\partial z}\right)\right] \\
& +\frac{1}{2} A^{2}\left[\left(\frac{-\beta}{k^{2}}+\frac{2 \beta k^{2}}{k^{4}}\right) \frac{\partial}{\partial x} k^{2}+\frac{2 \beta k \ell}{k^{4}} \frac{\partial}{\partial y} k^{2}+\frac{1}{\widehat{s}} \frac{2 \beta k m}{K^{4}} \frac{\partial}{\partial z} k^{2}\right] \\
& -\frac{1}{2} \frac{\beta k A^{2}}{k^{2}}\left[\frac{\partial k}{\partial x}+\frac{\partial \ell}{\partial y}+\frac{1}{\hat{s}} \frac{\partial m}{\partial z}\right] .
\end{aligned}
$$

Equation (4.17) is more useful than (4.15) because there are no time derivatives on the right hand side. It turns out that the right hand side of $(4.17)$ is related to the divergence of the group velocity $C$. Starting from $(4.12 a)-(4.12 c)$,

$$
\begin{aligned}
& \rightarrow \overrightarrow{\nabla_{3}} \cdot \vec{C}=\frac{\partial C}{\partial x}+\frac{\partial C}{\partial y}+\frac{\partial C_{z}}{\partial z}= \\
& \frac{\partial \bar{u}}{\partial x}+\frac{\beta}{K^{4}} \frac{\partial}{\partial x}\left(K^{2}\right)-\frac{4 \beta k^{2}}{k^{6}} \frac{\partial}{\partial x}\left(K^{2}\right)+\frac{2 \beta}{k^{4}} \frac{\partial}{\partial x}\left(k^{2}\right)+\frac{\partial v}{\partial y}-\frac{4 \beta k l}{k^{6}} \frac{\partial}{\partial y}\left(K^{2}\right) \\
&+\frac{2 \beta}{k^{4}} \frac{\partial}{\partial y}(k l)-\frac{4 \beta k m}{\widehat{S} k^{6}} \frac{\partial}{\partial z}\left(k^{2}\right)+\frac{2 \beta}{\widehat{S} k^{4}} \frac{\partial}{\partial z}(k m) .
\end{aligned}
$$


Multiplying the LHS of (4.18) by $\mathrm{E}$ and the RHS by its. equivalent, via (2.16), $\frac{1}{4} \mathrm{~A}^{2} \mathrm{~K}^{2}$

E $\overrightarrow{\nabla_{3}} \cdot \vec{C}=\underbrace{\frac{1}{4} A^{2} K^{2}\left(\frac{\partial \vec{u}}{\partial x}+\frac{\partial \vec{v}}{\partial y}\right)}+\frac{1}{4} \frac{A^{2} \beta}{K^{2}} \frac{\partial}{\partial x}\left(K^{2}\right)-\frac{\beta k A^{2}}{K^{4}}\left[k \frac{\partial}{\partial x}\left(K^{2}\right)\right.$

(1)

$\left.+\ell \frac{\partial}{\partial y}\left(k^{2}\right)+\frac{1}{S} m \frac{\partial}{\partial z}\left(k^{2}\right)\right]+\underbrace{\frac{1}{2} \frac{\beta A^{2}}{k^{2}}\left[\frac{\partial}{\partial x}\left(k^{2}\right)+\frac{\partial}{\partial y}(k \ell)+\frac{1}{\hat{s}} \frac{\partial}{\partial z}(k m)\right]}$.

(2)

We now assume that the mean flow is non-divergent:

$$
\frac{\partial \bar{u}}{\partial x}+\frac{\partial \bar{v}}{\partial y}=0
$$

Then term (1) in (4.19) vanishes. Term (2)an be simplified using identities relating the slow changes of wavenumber components which are derived from the fundamental definition of wavenumber given in $(3.5 b)$, namely

$$
\frac{\partial}{\partial y}(k \ell)=k \frac{\partial \ell}{\partial y}+\ell \frac{\partial k}{\partial y}=k \frac{\partial \ell}{\partial y}+\ell \frac{\partial^{2} \alpha}{\partial y \partial x}=k \frac{\partial \ell}{\partial y}+\ell \frac{\partial \ell}{\partial x},
$$

and

$$
\frac{\partial}{\partial z}(k m)=k \frac{\partial m}{\partial z}+m \frac{\partial k}{\partial z}=k \frac{\partial m}{\partial z}+m \frac{\partial^{2} \alpha}{\partial z \partial x}=k \frac{\partial m}{\partial z}+m \frac{\partial m}{\partial x} .
$$

With $(4.21 a)$ and $(4.21 b)$, term 2 in $(4.19)$ becomes

$$
\begin{aligned}
\text { (2) } & =\frac{1}{2} \frac{\beta A^{2}}{K^{2}} k\left[\frac{\partial k}{\partial x}+\frac{\partial \ell}{\partial y}+\frac{1}{\widehat{S}} \frac{\partial m}{\partial z}\right]+\frac{\beta A^{2}}{2 K^{2}}\left[k \frac{\partial k}{\partial x}+\ell-\frac{\partial \ell}{\partial x}+\frac{1}{\widehat{S}}-\frac{\partial m}{\partial x}\right] \\
& =\frac{B A^{2}}{2 K^{2}} k\left[\frac{\partial k}{\partial x}+\frac{\partial \ell}{\partial y}+\frac{1}{\widehat{S}} \frac{\partial m}{\partial z}\right]+\frac{\beta A^{2}}{4 K^{2}} \frac{\partial}{\partial x}\left(K^{2}\right) .
\end{aligned}
$$

Using (4.22), (4.19) finally becomes 
E $\stackrel{\vec{\nabla}}{\vec{\nabla} \cdot C}=\frac{\beta A^{2}}{2 K^{2}} k\left[\frac{\partial k}{\partial x}+\frac{\partial \ell}{\partial y}+\frac{1}{\widehat{S}} \frac{\partial m}{\partial z}\right]+\frac{\beta A^{2}}{2 K^{2}} \frac{\partial}{\partial x}\left(K^{2}\right)$

$$
-\frac{\beta k A^{2}}{K^{4}}\left[k \frac{\partial}{\partial x}\left(K^{2}\right)+\ell \frac{\partial}{\partial y}\left(K^{2}\right)+\frac{1}{\widehat{S}} m \frac{\partial}{\partial z}\left(K^{2}\right)\right],
$$

which can be used to rewrite $(4.17)$ as

$$
\begin{aligned}
\frac{D E}{D t}= & -E \nabla_{3} \cdot C+\frac{1}{2} A^{2}\left[k\left(k \frac{\partial \bar{u}}{\partial x}+\ell \frac{\partial \bar{v}}{\partial x}\right)+\ell\left(k \frac{\partial \bar{u}}{\partial y}+\ell \frac{\partial \bar{v}}{\partial y}\right)\right. \\
& \left.+\frac{1}{\widehat{S}} m\left(k \frac{\partial \bar{u}}{\partial z}+\ell \frac{\partial \bar{v}}{\partial z}\right)\right] .
\end{aligned}
$$

Remembering the definition of $\frac{D}{D t}$ (given in (3.11)), an equivalent form is

$$
\begin{aligned}
\frac{\partial E}{\partial t}+\nabla_{3} \cdot(C E)=\frac{1}{2} A^{2}\left[k \left(k \frac{\partial \bar{u}}{\partial x}+\ell \frac{\partial \bar{v}}{\partial x}\right.\right. & +\ell\left(k \frac{\partial \bar{u}}{\partial y}+\ell \frac{\partial \bar{v}}{\partial y}\right) \\
& \left.+\frac{1}{\widehat{S}} m\left(k \frac{\partial \bar{u}}{\partial z}+\ell \frac{\partial \bar{v}}{\partial z}\right)\right]
\end{aligned}
$$

This is one of the important results in this article. It states that in the absence of mean flow gradients, the wave energy density satisfies a local conservation law giving the rate of change of energy in an arbitrary volume in balance with the flow of energy into or out of that volume, with the appropriate flow velocity being the group velocity.

In the presence of mean flow variations, wave energy is not conserved. We must turn to wave action and wave enstrophy to find conservation laws which hold in the presence of mean flow variations.

4b. Conservation laws for wave action and wave enstrophy It turns out to be convenient to define a generalized wave action $\widehat{A}$ by 


$$
\widehat{A}=\frac{f(k) E}{\omega^{\prime}},
$$

where $f(k)$ is an arbitrary function of the wavenumber component $k$, and the intrinsic frequency $\omega^{-}$is given (by (3.23) and (4.9b)) by

$$
\omega^{\prime}=\frac{-\beta k}{k^{2}} \text {, }
$$

whence

$$
\widehat{A}=\frac{-f(k) E K^{2}}{\beta k}
$$

Operating on both sides with $\frac{\mathrm{D}}{\mathrm{Dt}}$,

$\frac{D \hat{A}}{D t}=\frac{-f(k) K^{2}}{B k} \frac{D E}{D t}-\frac{E K^{2}}{\beta k} \frac{D f(k)}{D t}+\frac{f(k) E K^{2}}{B k^{2}} \frac{D k}{D t}-\frac{f(k) E}{B k} \frac{D}{D t}\left(K^{2}\right)$.

Noting that $\frac{D}{D t} f(k)=\frac{\partial f}{\partial k} \frac{D k}{D t}$, and using $(3.27 a)-(3.27 c)$,

$\frac{D \hat{A}}{D t}=\frac{-f(k) K^{2}}{B k} \frac{D E}{D t}+\left[\frac{-E K^{2}}{B k} \frac{\partial f}{\partial k}+\frac{f(k) E K^{2}}{B k^{2}}\right] \frac{D k}{D t}$

$$
\begin{gathered}
\frac{-f(k) E}{\beta k} 2\left[k \frac{D k}{D t}+\ell \frac{D \ell}{D t}+\frac{1}{\widehat{S}} m \frac{D m}{D t}\right] \\
=\frac{-f(k) K^{2}}{\beta k} \frac{D E}{D t}+\frac{E K^{2}}{\beta k}\left[\frac{f}{k}-\frac{\partial f}{\partial k}\right] \cdot\left[-k \frac{\partial \vec{u}}{\partial x}-\ell \frac{\partial \vec{v}}{\partial x}\right] \\
+\frac{f(k) E}{B k} \cdot 2 \cdot\left[k\left(k \frac{\partial \bar{u}}{\partial x}+\ell \frac{\partial \bar{v}}{\partial x}\right)+\ell\left(k \frac{\partial \vec{u}}{\partial y}+\ell \frac{\partial \bar{v}}{\partial y}\right)\right. \\
\left.+\frac{1}{\widehat{s}} m\left(k \frac{\partial \bar{u}}{\partial z}+\ell \frac{\partial \bar{v}}{\partial z}\right)\right] .
\end{gathered}
$$

Using $(4.24 a),(4.29)$ becomes 
$\frac{\widehat{D A}}{D t}=\frac{f(k) K^{2} E}{\beta k} \overrightarrow{\nabla_{3}} \cdot \vec{C}+\left[\frac{-f K^{2}}{\beta k} \frac{1}{2} A^{2}+\frac{2 f E}{\beta k}\right] \cdot\left[k\left(k \frac{\partial \bar{u}}{\partial x}+\ell \frac{\partial \bar{v}}{\partial y}\right)\right.$

$\left.+\ell\left(k \frac{\partial \bar{u}}{\partial y}+\ell \frac{\partial \bar{v}}{\partial y}\right)+\frac{1}{\hat{S}} m\left(k \frac{\partial \vec{u}}{\partial z}+\ell \frac{\partial \bar{v}}{\partial z}\right)\right]$

$$
+\frac{E K^{2}}{\beta k}\left[\frac{f}{k}-\frac{\partial f}{\partial k}\right] \cdot\left[-k \frac{\partial \bar{u}}{\partial x}-\ell \frac{\partial \bar{v}}{\partial x}\right] .
$$

Equation (4.30) can be simplified considerably by using the expressions for $\mathrm{E}$ given by $(2.10)$ and for $\widehat{A}$ by $(4.27)$ :

$\frac{\widehat{D A}}{D t}=-\hat{A} \nabla_{3} \cdot \vec{C}+\frac{E K^{2}}{\beta k}\left[\frac{f}{k}-\frac{\partial f}{\partial k}\right]\left[-k \frac{\partial \bar{u}}{\partial x}-\ell \frac{\partial \bar{v}}{\partial x}\right]$.

Two choices of $f(k)$ are of particular interest.

1) $f(k)=1$.

Then $\widehat{A} \equiv \widehat{A}(1)=\frac{E}{\omega^{-}}$is wave action (Andrews and McIntyre, 1978),

$$
\widehat{A}(1)=-\frac{A^{2} K^{4}}{4 B k}
$$

For this choice of $\mathrm{f},(4.31)$ becomes

$$
\frac{\partial \hat{A}^{(1)}}{\partial t}+\overrightarrow{\nabla_{3}} \cdot\left(\widehat{A}^{(1)} \vec{C}\right)=\widehat{A}^{(1)} \frac{1}{k}\left[k \frac{\partial \bar{u}}{\partial x}+\ell \frac{\partial \bar{v}}{\partial x}\right]
$$

This important result (also given by Young and Rhines, 1980), states that even when the mean flow $(\bar{u}, \bar{v})$ depends on $y$ and $z$, wave action is conserved locally, with the relevant flow of wave action occurring with the group velocity.

2) $f(k)=-\beta k$, whence

$$
\widehat{A} \equiv \widehat{A}(2)=E K^{2}=P \text {, }
$$

so that $\widehat{A}^{(2)}$ is the wave potential enstrophy density given in (2.19). 
From $(4.31)$,

$$
\frac{\widehat{\partial A}}{\partial t}-\overrightarrow{\left.\nabla^{2}\right)}+\nabla_{3} \cdot\left(\widehat{A}^{(2)} \cdot \vec{C}\right)=0
$$

$(4.34)$

This result, (again given by Young and Rhines, 1980) states that wave enstrophy is conserved locally for arbitrary (slow) variations of the mean flow ( $\vec{u}, \vec{v})$. 
5. Connection with Wave Activity, Potential Vorticity and the Eliassen-Palm

\section{F1ux; Alternative Derivations}

5a. Eliassen-Palm Flux Vector

The Eliassen-Palm flux vector $\vec{F}=\left(F_{y}, F_{z}\right)$ can be expressed (see the Appendix) as :

$$
\begin{aligned}
& F_{y}=e^{-z / H} \frac{\partial \psi}{\partial y} \frac{\partial \psi}{\partial x}, \\
& F_{z}=e^{-z / H} \frac{1}{\widehat{S}} \frac{\partial \psi}{\partial x} \frac{\partial \psi}{\partial z}
\end{aligned}
$$

Here the primes denote perturbations from the zonal mean, and the overbars a zonal average. From our basic solution (2.10) for a Rossby wave,

or

$$
\begin{aligned}
& F_{y}=\frac{1}{2} A^{2} k l, \\
& F_{z}=\frac{1}{\hat{S}} \frac{1}{2} A^{2} k m, \\
& \vec{F}=\frac{1}{2} A^{2}\left(k l, \frac{1}{\hat{S}} k m\right):
\end{aligned}
$$

Now define

$$
\widehat{A}(3) \equiv \frac{1}{B} P=\frac{1}{4 B} A^{2} K^{4}=-k \hat{A}^{(1)},
$$

and write down the intrinsic group velocity from $(4.12 a)-(4.12 c)$ :

$$
\overrightarrow{c^{\prime}}=\left(C_{x}^{\prime}, \frac{2 \beta k \ell}{\mathrm{K}^{4}}, \frac{2 \beta k m}{\hat{S K}^{4}}\right) \text {. }
$$

From $(5.2)-(5.4)$,

$$
\overrightarrow{C^{\prime}} \widehat{A}^{(3)}=\left(C_{x}^{-} \widehat{A}^{(3)}, \frac{1}{2} \widehat{A}^{2} k \ell, \frac{1}{2} \frac{1}{\widehat{S}} A^{2} k m\right),
$$


or

$$
\left(C^{-}-\hat{A}^{(3)}\right)_{\perp}=\vec{F}
$$

Where $\perp$ denotes a projection onto the $y-z$ plane. Thus, the intrinsic group velocity times the wave enstrophy, divided by $B$, gives the Eliassen-Palm flux

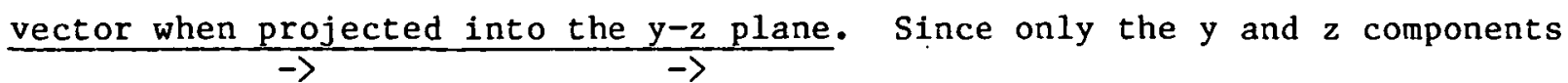
enter (5.5), $\mathrm{C}^{-}$is identical to $\mathrm{C}$ if the basic state meridional velocity $\overline{\mathrm{v}}$ vanishes.

$5 b$. Conservation of wave activity in the case of homogeneity in the $x$ direction. When $\bar{v}=0$ and the wave amplitude and wavenumber $(k, \ell, m)$ are independent of $x$, it is possible to derive a conservation law for wave activity directly from equation (2.9), without explicit consideration of the variation of $A$ and ( $k, l, m)$ in $y$ and $z$. We start by multiplying (2.9) by $\frac{1}{\beta} q^{-}$and zonally averging:

$$
\frac{\partial}{\partial t}\left(\frac{1}{2 \beta} \overline{\left(q^{\prime}\right)^{2}}\right)+\overline{v^{\prime} q^{\prime}}=0
$$

From $(2.6 \mathrm{~b})$,

$\overline{v^{\prime} q^{\prime}}=\overline{\frac{\partial \psi^{\prime}}{\partial x} \frac{\partial^{2} \psi^{\prime}}{\partial x^{2}}}+\overline{\frac{\partial \psi^{\prime}}{\partial x} \frac{\partial^{2} \psi^{\prime}}{\partial y^{2}}}+\frac{1}{\frac{1}{S}} e^{z / H} \overline{\frac{\partial \psi^{\prime}}{\partial x} \frac{\partial}{\partial z}\left(e^{-z / H} \frac{\partial \psi^{\prime}}{\partial z}\right)}$

$=\frac{\partial}{\partial y}\left(\overline{\frac{\partial \psi^{\prime}}{\partial x}} \frac{\partial \psi^{\prime}}{\partial y}\right)+\frac{1}{\hat{S}} e^{z / H} \frac{\partial}{\partial z}\left(e^{-z / H} \frac{\partial \psi^{\prime}}{\partial z} \frac{\partial \psi^{\prime}}{\partial x}\right)$

$=\left(\right.$ using (5.1a), (5.1b)) $e^{z / H} \frac{\partial}{\partial y} F_{y}+e^{z / H} \frac{\partial}{\partial z} F_{z}$,

where we have used the fact thst $\widehat{S}$ is assumed constent in $z$. Using (5.5), (5.7) becomes

$$
\overline{v^{\prime} q^{\prime}}=\overrightarrow{\nabla_{\perp}} \cdot\left(e^{z / H} \vec{C} \widehat{A}^{(3)}\right)
$$


where $\vec{C}$ can be used instead of $\overrightarrow{c^{-}}$because $\bar{v}=0$, and $\overrightarrow{\nabla_{\perp}}$ denotes the operator $\left(\frac{\partial}{\partial y}, \frac{\partial}{\partial z}\right)$. Equation (5.8) relates the flux of potential vorticity to the divergence of the group velocity times a quantity $\mathrm{e}^{z / H} \widehat{A}^{(3)}$ related to wave enstrophy.

Now, from (2.11), our basic solution (2.9) gives

$$
\frac{1}{2 \beta}\left(\overline{\left.q^{0}\right)^{2}}=\frac{1}{4 \beta} K^{4} A^{2} e^{z / H}=\widehat{A}^{(3)} e^{z / H},\right.
$$

so we define $\widehat{A}^{(4)}$ by

$$
\widehat{A}(4) \equiv \mathrm{e}^{z / \hat{H}_{A}}(3)=\mathrm{e}^{z / H} \frac{1}{B} \mathrm{P}=\frac{1}{4 \beta} \mathrm{K}^{4} \mathrm{~A}^{2} \mathrm{e}^{z / H}=\frac{1}{2 \beta}\left(\overline{\left.\mathrm{q}^{\prime}\right)^{2}}\right. \text { (5.9) }
$$

From (5.8) and $(5.9),(5.6)$ becomes

$$
\frac{\partial}{\partial t} \widehat{A}^{(4)}+\vec{\nabla}_{\perp} \cdot\left(\vec{C} \widehat{A}^{(4)}\right)=0
$$

$\widehat{\mathrm{A}}^{(4)}$ is referred to by Edmon et al. (1980) as wave activity. Note that the local conservation law (5.10) only involves the two dimensional divergence $\overrightarrow{\nabla_{\perp}} \equiv\left(\frac{\partial}{\partial y}, \frac{\partial}{\partial z}\right)$ rather than the three dimensional form in, for example, (4.34). This is because $A^{(4)}$ and $C$ are both independent of $x$ by assumption. It is interesting that this derivation does not involve any explicit calculation of just how $\vec{C}$ and $\widehat{A}^{(4)}$ depend an $y$ and $z$, and in particular how $A$ and $(k, \ell, m)$ vary in space. It is similar in this regard to a derivation of (4.34) presented by Hoskins et al. (1983). 
1. Derivation of the quasigeostrophic potential vorticity equation in $z$ coordinates.

Our starting point is the quasigeostrophic equations in pressure coordinates, with constant static stablitity, from Lorenz (1960):

$$
\begin{aligned}
& \frac{\partial \theta}{\partial t}=-J(\psi, \theta)-\omega \frac{\partial \theta_{s}}{\partial \mathrm{p}} \\
& \frac{\partial}{\partial t}\left(\nabla_{2}^{2} \psi\right)=-J\left(\psi, \nabla_{2}^{2} \psi\right)-J(\psi, \mathrm{f})-\nabla_{2} \cdot\left(\mathrm{f} \mathrm{v}_{\mathrm{D}}\right) \\
& \mathrm{g} \nabla_{2^{*}}^{2}=\nabla_{2} \cdot\left(\begin{array}{ll}
\mathrm{f} & -> \\
z_{2}
\end{array}\right) .
\end{aligned}
$$

In these equations, $\theta$ is potential temperature, $\theta_{S}$ the reference state potential temperature (depending on $\mathrm{p}$ only), $\psi$ is the geostrophic streamfunction,

$\overrightarrow{\nabla_{2}}$ the horizontal gradient operator and $\nabla_{2}^{2}$ the horizontal Laplacian, $\nabla_{2}^{2} \psi$

the vorticity, $\omega=\frac{d p}{d t}, z^{*}$ is altitude and $f=2 \Omega \sin \phi$, where $\Omega$ is the angular rotation rate of the earth and $\phi$ the latitude. $J(A, B)$ represents the Jacobian of the variables $\mathrm{A}$ and $\mathrm{B}$. Also,

$$
\begin{array}{ll}
\nabla_{2} & -v_{D}
\end{array}+\frac{\partial \omega}{\partial p}=0 .
$$

$\rightarrow$

where $v_{D}$ is the divergent part of the wind.

Neglecting the effects of spherical geometry, and neglecting the latitudinal variation of $f$ except in the term $J(\psi, f)$ in $(A .2),(A .1)-(A .3)$ become

$$
\begin{gathered}
\frac{\partial \theta}{\partial t}+\frac{\partial \psi}{\partial x} \frac{\partial \theta}{\partial y}-\frac{\partial \psi}{\partial y} \frac{\partial \theta}{\partial x}=-\omega \frac{\partial \theta_{s}}{\partial p} \\
\frac{\partial}{\partial t}\left(\nabla_{2}^{2} \psi\right)+\frac{\partial \psi}{\partial x} \frac{\partial\left(\nabla_{2}^{2} \psi\right)}{\partial y}-\frac{\partial \psi}{\partial y} \frac{\partial\left(\nabla_{2}^{2} \psi\right)}{\partial x}+\beta \frac{\partial \psi}{\partial x}-\mathrm{f}_{\mathrm{o}} \frac{\partial \omega}{\partial \mathrm{p}}=0 \\
\overline{\mathrm{g}} \bar{z}^{\star}=\mathrm{f}_{\mathrm{o}}^{-} \psi^{-} \\
-32-
\end{gathered}
$$


In these equations $\mathrm{y}$ is the northward distance and $\mathrm{x}$ the eastward distance on $a$ plane targent to the earth (the so-called "beta-plane") at latitude $\phi_{0}$. (For a rigorous derivation of this approximation, see Chapter 6 of Pedlosky, 1979): $f$ has been approximated in $(A .6)$ as $f=f_{o}+B y$, where $f_{o}=2 \Omega \sin \phi_{0}$. In (A.7) $f$ is taken simply as $\mathrm{f}_{\mathrm{o}}$.

Introducing the new vertical coordinate (as in Section 2 ).

$$
z=-H \ln \left(p / p_{0}\right),
$$

where $\mathrm{H}$ is the scale height, and $\mathrm{p}_{\mathrm{O}}=1000 \mathrm{mb}$, we have the identities

$$
\frac{\partial}{\partial p}=\frac{\partial z}{\partial p} \frac{\partial}{\partial z}=\frac{-H}{p} \frac{\partial}{\partial z}=\frac{-H}{p_{O}} e^{z / H} \frac{\partial}{\partial z}
$$

and

$$
\omega=\frac{d p}{d t}=\frac{\partial p}{\partial z} \frac{d z}{d t}=\frac{\partial p}{\partial z} w=\frac{-p_{0}}{H} e^{-z / H} w
$$

where $w \equiv \frac{\mathrm{d} z}{\mathrm{dt}}$ is the vertical velocity in $\mathrm{z}$ coordinates.

Using the equation of state $\mathrm{p} \alpha=\mathrm{RT}$, where $\alpha$ is the volume per unit mass and $R$ the gas constant, and the hydrostatic balance $\frac{\partial z}{\partial p}{ }^{*}=\frac{-\alpha}{g}$, where $g$ is the gravitational acceleration, we have

$$
\mathrm{g} \frac{\partial z^{*}}{\partial \mathrm{p}}=-\alpha=\frac{-\mathrm{RT}}{\mathrm{p}}
$$

Hence (A.7) becomes

$$
T=\frac{-p}{R} f_{0} \frac{\partial \psi}{\partial p}
$$

or

$$
T=\frac{H f}{R} o \frac{\partial \psi}{\partial z}
$$

We convert (A.5) to a prognostic equation for temperature by multiplying it by $\left(\frac{\mathrm{p}}{\mathrm{p}_{\mathrm{o}}}\right)^{K}$, where $k=\mathrm{R} / \mathrm{C}_{\mathrm{p}}, \mathrm{C}_{\mathrm{p}}$ being the specific heat at constant pressure: 


$$
\frac{\partial T}{\partial t}+\frac{\partial \psi}{\partial x} \cdot \frac{\partial T}{\partial y}-\frac{\partial \psi}{\partial y} \frac{\partial T}{\partial x}=-\omega\left(\frac{p}{P_{0}}\right) \kappa \frac{\partial \theta_{s}}{\partial p}
$$

Further,

$$
-\omega\left(\frac{\mathrm{p}}{p_{\mathrm{o}}}\right)^{\kappa} \frac{\partial \theta_{s}}{\partial \mathrm{p}}=-\omega\left(\frac{\mathrm{p}}{\mathrm{p}_{\mathrm{o}}}\right)^{\kappa} \frac{\mathrm{d} \theta_{s}}{\mathrm{dp}}=-\omega \frac{\partial z}{\partial \mathrm{p}}\left[\left(\frac{\mathrm{p}}{\mathrm{p}_{\mathrm{o}}}\right)^{\kappa} \frac{\mathrm{d} \theta_{s}}{\mathrm{dz}}\right] .
$$

Defining (as in Section 2)

$$
S \equiv\left(\frac{p}{p_{0}}\right) \kappa \frac{d \theta_{s}}{d z},
$$

and using (A.10), we have

$$
-\omega\left(\frac{p}{P_{0}}\right)^{\kappa} \frac{\partial \theta_{s}}{\partial p}=-w S \text {, }
$$

so that (A.13) becomes

$$
\frac{\partial T}{\partial t}+\frac{\partial \psi}{\partial x} \frac{\partial T}{\partial y}-\frac{\partial \psi}{\partial y} \frac{\partial T}{\partial x}=-w S
$$

Finally, the term $-f_{o} \frac{\partial \omega}{\partial p}$ in (A.6) must be rewritten using (A.9) and (A.10):

$-f_{0} \frac{\partial \omega}{\partial p}=-f_{0} \frac{\partial}{\partial p}\left[\frac{\partial p}{\partial z} \cdot w\right]=-f_{0} \frac{\partial z}{\partial p} \frac{\partial}{\partial z}\left[\frac{\partial p}{\partial z} \cdot w\right]=-f_{0} e^{z / H} \frac{\partial}{\partial z}\left[e^{-z / H} w\right]$,

whence (A.6) becomes:

$$
\frac{\partial}{\partial t}\left(\nabla_{2}^{2} \psi\right)+\frac{\partial \psi}{\partial x} \frac{\partial}{\partial y}\left(\nabla_{2}^{2} \psi\right)-\frac{\partial \psi}{\partial y} \frac{\partial}{\partial x}\left(\nabla_{2}^{2} \psi\right)+\beta \frac{\partial \psi}{\partial x}=f_{0} e^{z / H} \frac{\partial}{\partial z}\left[e^{-z / H_{-}}\right]=0
$$

The conservation law for (quasigeostrophic) potential vorticity is derived from (A.12), (A.15) and (A.17):

$$
w=\frac{1}{S}\left\{-\frac{\partial T}{\partial t}-\frac{\partial \psi}{\partial x} \frac{\partial T}{\partial y}+\frac{\partial \psi}{\partial y} \frac{\partial T}{\partial x}\right\},
$$

which, using (A.12) becomes 
$\mathbf{w}=\frac{\mathbf{f}_{\mathrm{O}}}{\mathrm{R} S}\left\{-\frac{\partial^{2} \psi}{\partial t \partial z}-\frac{\partial \psi}{\partial x} \frac{\partial^{2} \psi}{\partial z \frac{\psi}{\partial y}}+\frac{\partial \psi}{\partial y} \frac{\partial^{2}}{\partial z} \frac{\psi}{\partial x}\right\}$.

Combining (A.17) and (A.18) gives, (again treating $S$ as a constant):

$$
\begin{aligned}
& \frac{\partial}{\partial t}\left(\nabla_{2}^{2} \psi\right)+\frac{\partial \psi}{\partial x} \frac{\partial}{\partial y}\left(\nabla_{2}^{2} \psi\right)-\frac{\partial \psi}{\partial y} \frac{\partial}{\partial x}\left(\nabla_{2}^{2} \psi\right)+\beta \frac{\partial \psi}{\partial x} \\
& -\underset{\mathrm{RS}}{\mathrm{f}_{\mathrm{O}}{ }^{2} \mathrm{H}} \mathrm{e}^{\mathrm{z} / \mathrm{H}} \frac{\partial}{\partial z}\left\{\mathrm{e}^{-z / H}\left[-\frac{\partial^{2} \psi}{\partial z \partial t}-\frac{\partial \psi}{\partial \mathrm{x}} \frac{\partial^{2}}{\partial z} \frac{\psi}{\partial y}+\frac{\partial \psi}{\partial y} \frac{\partial^{2} \psi}{\partial z \partial x}\right]\right\}=0 .
\end{aligned}
$$

We write the last term in (A.19) as a sum of three terms:

$$
\begin{aligned}
& \underset{\mathrm{RS}}{\mathrm{f}_{\mathrm{O}}{ }^{2} \mathrm{H}}\left[\mathrm{e}^{\mathrm{z} / \mathrm{H}} \frac{\partial}{\partial z}\left\{\mathrm{e}^{-z / \mathrm{H}} \frac{\partial^{2} \psi}{\partial z \partial t}\right\}+\mathrm{e}^{z / H} \frac{\partial}{\partial z}\left\{\mathrm{e}^{-z / H} \frac{\partial \psi}{\partial x} \frac{\partial^{2} \psi}{\partial z \partial y}\right\}\right.
\end{aligned}
$$

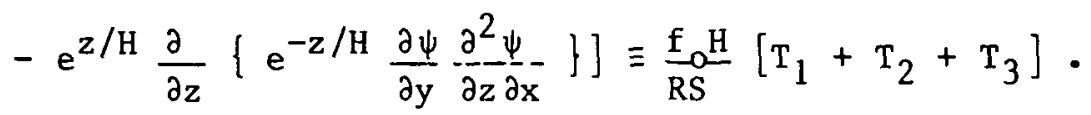

$$
\begin{aligned}
& \mathrm{T}_{1}=\frac{\partial}{\partial t}\left\{\mathrm{e}^{\mathrm{z} / \mathrm{H}} \frac{\partial}{\partial z}\left(\mathrm{e}^{-\mathrm{z} / \mathrm{H}} \frac{\partial \psi}{\partial z}\right)\right\}, \\
& \mathrm{T}_{2}=\frac{\partial \psi}{\partial x} e^{z / H} \frac{\partial}{\partial z}\left\{e^{-z / H} \frac{\partial^{2} \psi}{\partial z \partial y}\right\}+\frac{\partial^{2} \psi}{\partial z \partial y} \cdot \frac{\partial^{2} \psi}{\partial z} \frac{\psi}{\partial x} \\
& =\frac{\partial \psi}{\partial x} \frac{\partial}{\partial y}\left[e^{z / H} \frac{\partial}{\partial z}\left(e^{-z / H} \frac{\partial \psi}{\partial z}\right)\right]+\frac{\partial^{2} \psi}{\partial z \partial y} \cdot \frac{\partial^{2} \psi}{\partial z \partial x} \\
& \mathrm{~T}_{3}=-\frac{\partial \psi}{\partial y} e^{z / H} \frac{\partial}{\partial z}\left\{e^{-z / H} \frac{\partial^{2} \psi}{\partial z \partial x}\right\}-\frac{\partial^{2} \psi}{\partial z \partial x} \cdot \frac{\partial^{2} \psi}{\partial z \partial y} \\
& =-\frac{\partial \psi}{\partial y} \frac{\partial}{\partial x}\left[e^{z / H} \frac{\partial}{\partial z}\left(e^{-z / H} \frac{\partial \psi}{\partial z}\right)\right]-\frac{\partial^{2} \psi}{\partial z} \frac{\psi}{\partial y} \cdot \frac{\partial^{2} \psi}{\partial z \partial x}
\end{aligned}
$$

Introducing the parameter $\widehat{\mathrm{S}}$ from equation (2.5) of the text, (A.19) through (A.21c) may be combined: 
$\frac{\partial}{\partial t}\left[\nabla_{2}^{2} \psi+\frac{1}{\hat{S}} e^{z / H} \frac{\partial}{\partial z}\left(e^{-z / H} \frac{\partial \psi}{\partial z}\right)\right]$

$+\frac{\partial \psi}{\partial y} \frac{\partial}{\partial x}\left[\nabla_{2}^{2} \psi+\frac{1}{\hat{s}} e^{z / H} \frac{\partial}{\partial z}\left(e^{-z / H} \frac{\partial \psi}{\partial z}\right)\right]$

$-\frac{\partial \psi}{\partial y} \frac{\partial}{\partial x}\left[\nabla_{2}^{2} \psi+\frac{1}{\hat{S}} e^{z / H} \frac{\partial}{\partial z}\left(e^{-z / H} \frac{\partial \psi}{\partial z}\right)\right] v+\beta \frac{\partial \psi}{\partial x}=0$.

This is just

$$
\left(\frac{\partial}{\partial t}-\frac{\partial \psi}{\partial y} \frac{\partial}{\partial x}+\frac{\partial \psi}{\partial x}-\frac{\partial}{\partial y}\right) q=0
$$

with

$$
q=f_{o}+\beta y+\nabla_{2}^{2} \psi+\frac{1}{\hat{S}} e^{z / H} \frac{\partial}{\partial z}\left(e^{-z / H} \frac{\partial \psi}{\partial z}\right)
$$

We have thus derived equations (2.2) and (2.3) in the text. 
2. Eliassen Palm Flux Vector in $\ln (p)$ coordinates

The Eliassen-Palm flux vector, in pressure coordinates, is given by Edmon et al. (1980) for the beta plane:

$$
\left.\overrightarrow{\mathrm{F}}=\left(\hat{\mathrm{F}}_{\mathrm{y}}, \hat{\mathrm{F}}_{\mathrm{p}}\right)=\left(-\overline{\mathrm{u}}^{-} \overline{\mathrm{v}}^{-}\right), \mathrm{f}_{\mathrm{o}} \frac{\overrightarrow{\mathrm{v}} \cdot \vec{\theta}}{\sigma}\right),
$$

where primes denote wave quantities, the overbar a zonal average, and $u, v$ are the geostrophic wind components: $u=\frac{-\partial \psi}{\partial y}, v=\frac{\partial \psi}{\partial x}$, while $\theta=T\left(\underline{p}_{0}\right) k$, where $T$ is given by (A.12). The static stability is given by $\sigma=\frac{\mathrm{d} \theta_{S}}{\mathrm{dp}}$, with $\theta_{S}$ the reference state potential temperature.

$$
\rightarrow
$$

To obtain $F$ in $z$ coordinates (see equation (2.1)), we work with the (twodimensional) divergence of $\overrightarrow{\widehat{F}}$ :

$$
\frac{\partial \hat{F}_{y}}{\partial y}+\frac{\partial \hat{F}}{\partial p}=+\frac{\partial}{\partial y}\left(\frac{\partial \psi}{\partial x} \frac{\partial \psi}{\partial y}\right)+\frac{\partial}{\partial p}\left(f_{o} \frac{\overrightarrow{v^{\prime} \theta^{\prime}}}{\sigma}\right)
$$

Using the definition of $S$ given in the text, and equation (A.9),

$$
\sigma=\frac{\mathrm{d} \theta_{S}}{\mathrm{dp}}=\frac{\mathrm{d} \theta_{S}}{\mathrm{dz}} \frac{\partial z}{\partial \mathrm{p}}=-\frac{\mathrm{d} \theta_{S}}{\mathrm{dz}} \frac{\mathrm{H}}{\mathrm{p}_{\mathrm{o}}} \mathrm{e}^{z / H}=-\mathrm{s}\left(\underline{\mathrm{p}}_{\mathrm{p}}\right)^{K} \frac{\mathrm{H}}{\mathrm{p}_{\mathrm{O}}} \mathrm{e}^{z / H},
$$

whence

$$
\begin{aligned}
& \frac{\partial}{\partial p}\left(f_{0} \frac{\overline{v^{\prime} \theta}}{\sigma}\right)=\left(-\frac{H}{p_{0}} e^{z / H}\right) \frac{\partial}{\partial z}\left(f_{0} \frac{\overrightarrow{v^{\prime} \theta^{\prime}}}{s}\left(\frac{p}{p_{0}}\right)^{K} \cdot\left(-\frac{p_{0}}{H}\right) e^{-z / H}\right) \\
& \left.=\mathrm{e}^{z / H} \frac{\partial}{\partial z} \underset{S}{\left(\underline{f}_{0}\right.} \overline{v^{\prime} T^{\prime}} e^{-z / H}\right)=(\text { using }(A .12)) e^{z / H} \frac{\partial}{\partial z}\left(\frac{1}{\hat{S}} \frac{\partial \psi^{\prime}}{\partial x} \frac{\partial \psi^{\prime}}{\partial z} e^{-z / H}\right) .
\end{aligned}
$$

Using (A.27),

$$
\begin{aligned}
& \frac{\partial \hat{F}_{y}}{\partial y}+\frac{\partial \widehat{F}_{p}}{\partial p}=e^{z / H}\left[\frac{\partial}{\partial y}\left(e^{-z / H} \overline{\frac{\partial \psi^{\prime}}{\partial x} \frac{\partial \psi^{-}}{\partial y}}\right)+\frac{\partial}{\partial z}\left(e^{-z / H} \frac{1}{\widehat{S} \frac{\partial \psi^{\prime}}{\partial x} \frac{\partial \psi^{\prime}}{\partial z}}\right)\right] \\
& \equiv e^{z / H}\left[\frac{\partial}{\partial y} F_{y}+\frac{\partial}{\partial z} F_{z}\right] .
\end{aligned}
$$

Equation (A.28) defines $\left(F_{y}, F_{z}\right)$ in the $z$ coordinate system. They are identical to $(5.1 \mathrm{a})$ and $(5.1 \mathrm{~b})$ of the text. 


\section{REFERENCES}

Andrews, D. G. and M. E. McIntyre, 1978: On wave action and its relatives. J. Fluid. Mech., 89, 647-664.

Bretherton, F. P. and C. J. R. Garrett, 1968: Wavetrains in inhomogeneous moving media. Proc. Roy. Soc. Lond. A., 302, 529-554.

Charney, J. G., 1947: On the scale of atmospheric motions. Geofys. Publ., 17, No. $2,1-17$.

Charney, J. G. and M. E. Stern, 1962: On the stability of internal baroclinic jets in a rotating atmosphere. J.Atmos. Sci., 19, 159-172.

Edmon, H. G., B. J. Hoskins and M. E. McIntyre, 1980: Eliassen-Palm cross sections for the troposphere. J.Atmos. Sci., 37, 2600-2616.

Hayes, W. D., 1970: Conservation of action and modal wave action. Proc. Roy. Soc. Lond. A., 320, 187-208.

Hoskins, B. J., I. N. James and G. H. White, 1983: The shape, propagation and mean-flow interaction of large-scale weather systems. J.Atmos. Sci., 40, 1595-1612.

Hoskins, B. J. and D. Karoly, 1981: The steady linear response of a spherical atmosphere to thermal and orographical forcing. J.Atmos Sci., 38, 1179-1196.

Karoly, D. J. and B. J. Hoskins, 1982: Three dimensional propagation of planetary waves. J.Met. Soc. Japan, 60, 109-123.

Lighthi11, J., 1978: Waves in Fluids, Cambridge University Press, Cambridge, 504 pp.

Lorenz, E. N., 1960: Energy and numerical weather prediction. Tellus, 12, 364-373.

Palmer, T. N., 1982: Properties of the Eliassen-Palm flux for planetary scale motions. J..Atmos. Sci., 39, 992-997.

Pedlosky, J., 1979: Geophysical Fluid Dynamics, Springer Verlag, New York, 624 pp.

Young, W. R. and P. B. Rhines, 1980: Rossby wave action, enstrophy and energy in forced mean flows. Geophys. Astrophys. Fluid Dyn., 15, 39-52. 


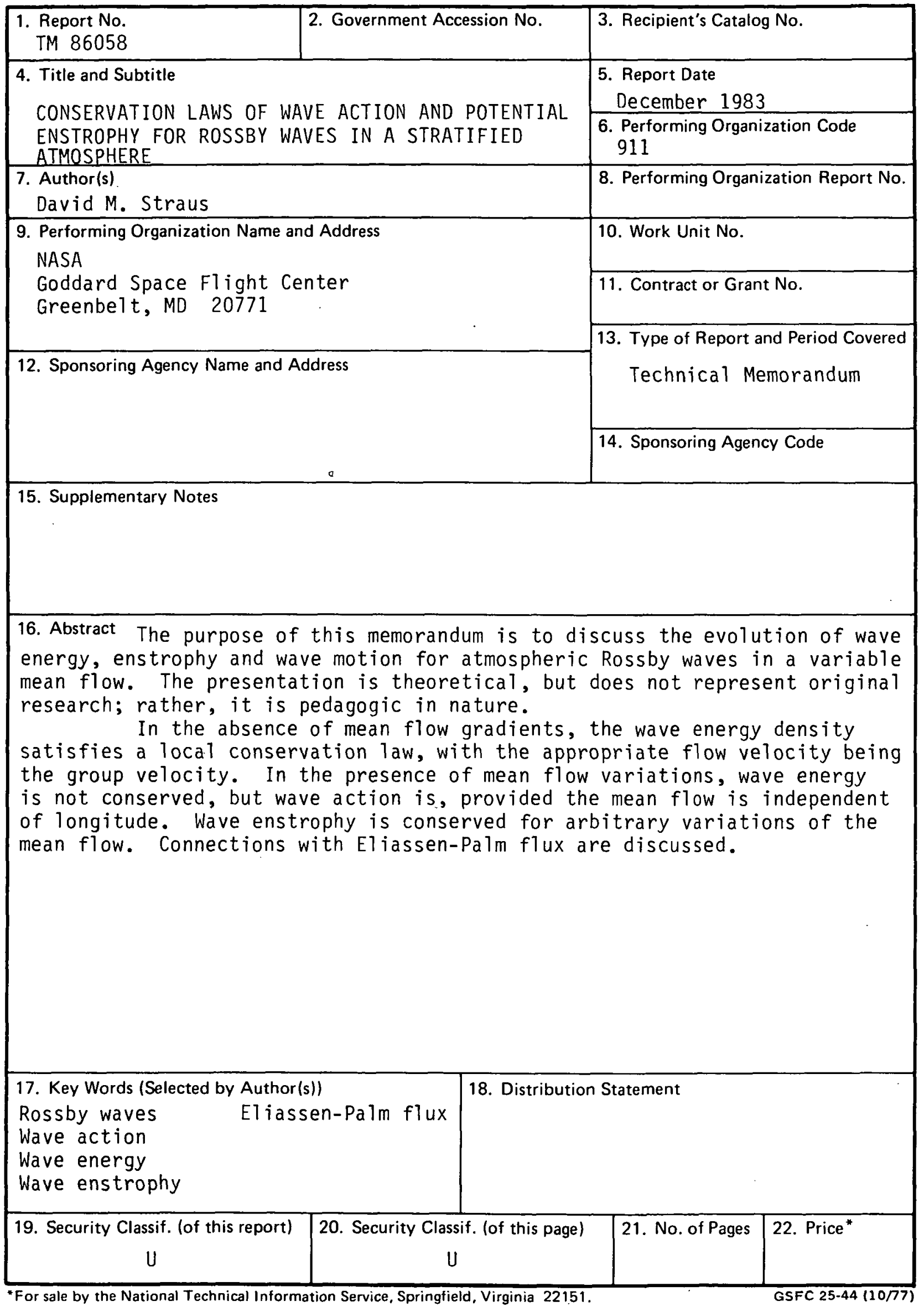

